\title{
A reduced Landau gyrofluid model for magnetic reconnection driven by electron inertia
}

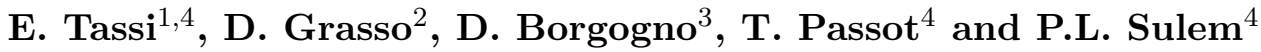 \\ ${ }^{1}$ Aix Marseille Univ, Université de Toulon, CNRS, CPT, Marseille, France \\ ${ }^{2}$ Istituto dei Sistemi Complessi - CNR and Dipartimento di Energia, Politecnico di Torino, \\ Torino 10129, Italy \\ ${ }^{3}$ Dipartimento di Energia, Politecnico di Torino, Torino 10129, Italy \\ ${ }^{4}$ Université Côte d'Azur, CNRS, Observatoire de la Côte d'Azur, Laboratoire J.L. Lagrange, \\ Boulevard de l'Observatoire, CS 34229, 06304 Nice Cedex 4, France
}

(Received $\mathrm{xx}$; revised $\mathrm{xx}$; accepted $\mathrm{xx}$ )

An electromagnetic reduced gyrofluid model for collisionless plasmas, accounting for electron inertia, finite ion Larmor radius effects and Landau fluid closures for the electron fluid is derived by means of an asymptotic expansion from a parent gyrofluid model. In the absence of terms accounting for Landau damping, the model is shown to possess a noncanonical Hamiltonian structure. The corresponding Casimir invariants are derived and use is made thereof, in order to obtain a set of normal field variables, in terms of which the Poisson bracket and the model equations take a remarkably simple form. The inclusion of perpendicular temperature fluctuations generalizes previous Hamiltonian reduced fluid models and, in particular, the presence of ion perpendicular gyrofluid temperature fluctuations reflects into the presence of two new Lagrangian invariants governing the ion dynamics. The model is applied, in the cold-ion limit, to investigate numerically a magnetic reconnection problem. The Landau damping terms are shown to reduce, by decreasing the electron temperature fluctuations, the linear reconnection rate and to delay the nonlinear island growth. The saturated island width, on the other hand, is independent on Landau damping. The fraction of magnetic energy converted into perpendicular kinetic energy also appears to be unaffected by the Landau damping terms, which, on the other hand, dissipate parallel kinetic energy as well as free energy due to density and electron temperature fluctuations.

\section{Introduction}

Reduced fluid models are a frequently adopted tool for the description of various phenomena in magnetized plasmas. In our context, reduced fluid models are those which apply to plasmas in the presence of a magnetic field with an intense component along one direction (strong guide field assumption) and are characterized by quadratic nonlinearities and strong scale anisotropy. Over the years, a large number of such models were derived, for instance for describing drift wave turbulence (Hasegawa \& Mima 1978; Hasegawa \& Wakatani 1983; Camargo et al. 1996), magnetic reconnection (Schep et al. 1994; Grasso et al. 2001; Borgogno et al. 2005; Del Sarto et al. 2006; Muraglia et al. 2009; Grasso \& Tassi 2015), fundamental aspects of nonlinear plasma dynamics in a tokamakrelevant ordering (Strauss 1976; Hazeltine et al. 1985, 1987), turbulence accounting for magnetic fluctuations parallel to the guide field direction, as of interest for the solar wind (Boldyrev et al. 2013; Schekochihin et al. 2009; Tassi et al. 2016), and ion temperature gradient driven modes (Kim et al. 1993), only to mention a very few examples. A 
number of reduced gyrofluid models, obtained from the evolution equations of moments of gyrokinetic distribution functions, were also derived in order to describe low-frequency plasma phenomena (see, e.g. Snyder \& Hammett (2001), Hammett et al. (1993), Dorland \& Hammett (1993), Brizard (1992), Beer \& Hammett (1996), Scott (2010), Waelbroeck \& Tassi (2012), Keramidas Charidakos et al. (2015) and Waelbroeck et al. (2009)). Gyrofluid models are indeed valid for phenomena with characteristic frequencies much smaller than the characteristic ion cyclotron frequency. A main feature of gyrofluid models is their accuracy in describing phenomena occurring on scales comparable with the ion thermal gyroradius. This distinguishes gyrofluid models from finite Larmor radius fluid models, which are those valid only on scales much larger than the ion thermal gyroradius (but which, on the other hand, possess the advantage of being formulated directly in terms of moments of particle distribution functions).

One of the main advantages of reduced fluid and gyrofluid models is their relatively little cost, in terms of computational resources, when compared to kinetic or gyrokinetic models. This becomes particularly evident in the case of numerical simulations where three spatial coordinates are involved. Also, the recurrent form of the nonlinearities in such models, characterized by a canonical Poisson bracket with two spatial coordinates as canonically conjugate variables, makes such models amenable to some analytical treatments which are often more complicated in more general, not reduced, fluid models. In particular, for many such reduced fluid and gyrofluid models, a noncanonical Hamiltonian structure was identifed (see, e.g. Tassi (2017) for a recent review), which allows, for instance, for the identification of conservation laws associated with Casimir invariants and the application of the Energy-Casimir method for stability analysis (see, e.g. Morrison (1998) and Marsden \& Ratiu (2002)). On the other hand, kinetic and gyrokinetic models offer a more complete description of the plasma behavior, accounting also for waveparticle interaction and generic (i.e. non-Maxwellian) equilibrium distribution functions. In order to reduce the gap suffered in comparison with kinetic or gyrokinetic models, a number of fluid and gyrofluid models were conceived, which retain kinetic effects, such as Landau damping, at least at the level of the linear theory. Various "Landau-fluid" models (not all of them "reduced" in the sense described above) were in particular derived (see, e.g. Hammett \& Perkins (1990), Dorland \& Hammett (1993), Snyder \& Hammett (2001), Hammett et al. (1992), Mattor \& Parker (1997), Mattor (1998), Passot \& Sulem (2004), Goswami et al. (2005) and Sulem \& Passot (2015)). The main application for such models was a more refined description of instabilities and turbulence in situations relevant for tokamaks and space plasmas. Among the further phenomena, where the application of Landau-fluid models can be relevant, is the investigation of kinetic effects on magnetic reconnection in collisionless plasmas. Indeed, in recent years, the impact on magnetic reconnection of closures taking into account Landau damping on a fluid model was investigated, focusing mainly on ion dynamics, by means of five and tenmoment fluid models (Wang et al. 2015; Ng et al. 2017). The importance of electron Landau damping in collisionless reconnection driven by electron inertia, on the other hand, was put in evidence by means of numerical simulations of a hybrid model treating the electron population with a drift-kinetic description (Loureiro et al. 2013). In such numerical simulations, dependence on only two spatial coordinates was assumed, whereas the original hybrid model (Zocco \& Schekochihin 2011) accounts for the dependence on the three spatial coordinates. In situations where the two-dimensional approximation is no longer valid, however, it is likely that reduced Landau-fluid models could offer a valid complementary tool to kinetic descriptions. The purpose of this article is, in the first place, to derive, by consistently following a prescribed ordering, a reduced Landaufluid model capable of describing magnetic reconnection driven by electron inertia. In 
its more general form, this reduced model accounts for a gyrofluid description of the ion species and for anisotropies in the temperature fluctuations. Electron Larmor radius effects, on the other hand, are neglected, which, together with the inclusion of electron inertia effects, leads to a regime where the parameter $\beta_{e}$, which is the ratio between the electron equilibrium pressure and the magnetic pressure exerted by the guide field, is of the order of the electron-to-ion mass ratio. Such low- $\beta_{e}$ regime, where Landau damping is in general significant, may be valid for laboratory as well as solar corona applications (Zocco \& Schekochihin 2011). Clearly, when compared to more general fluid models with kinetic effects such as those of Wang et al. (2015) and $\mathrm{Ng}$ et al. (2017), our reduced model sacrifices the range of applicability, in favor of simplicity. A further property of the model is that, in its non-dissipative limit (i.e. when Landau damping terms are absent), it possesses a Hamiltonian structure. The starting point for the derivation of the reduced Landau-gyrofluid model is a simplified version of the gyrofluid system described in Scott (2010). This model retains electron inertia, which is a necessary ingredient for the type of magnetic reconnection we intend to investigate. Also, the gyrofluid closures adopted in this model are such that they guarantee energy conservation in the non-dissipative limit. This property is desirable if one intends to derive a reduced model which is also energy-conserving, a necessary condition for having a Hamiltonian structure.

In the second place, we intend to apply the model to numerical simulations of collisionless reconnection triggered by a helically symmetric perturbation. Although this is not a fully three-dimensional case yet, it provides a simple, although non-trivial, case where the Landau damping terms present in the model can have an effect. Also, because we focus on the role of electron Landau damping terms, for simplicity, the cold-ion limit is considered in the simulations.

The paper is organized as follows. In Sec. 2 the reduced Landau-gyrofluid model is derived as an asymptotic expansion from a parent gyrofluid model. In Sec. 3 it is shown that the model, in its non-dissipative limit, possesses a noncanonical Hamiltonian structure and the corresponding Casimir invariants are given. In Sec. 4 the results of numerical simulations of magnetic reconnection in both the linear and nonlinear regime are described. We conclude in Sec. 5.

\section{Model equations}

\subsection{Parent gyrofluid model}

As starting point for the derivation of the model we consider the following gyrofluid system (Scott 2010), in normalized units, consisting of the evolution equations

$$
\begin{aligned}
& \frac{\partial N_{\alpha}}{\partial t}+\left[\Gamma_{1_{\alpha}} \varphi, N_{\alpha}\right]-\left[\Gamma_{1_{\alpha}} A_{\|}, U_{\alpha}\right]+\left[\Gamma_{2_{\alpha}} \varphi, T_{\perp_{\alpha}}\right]-\left[\Gamma_{2_{\alpha}} A_{\|}, Q_{\perp_{\alpha}}\right]+\frac{\partial U_{\alpha}}{\partial z}=0 \\
& \frac{\partial}{\partial t}\left(\frac{m_{\alpha}}{m_{i}} U_{\alpha}+\operatorname{sgn}\left(q_{\alpha}\right) \Gamma_{1_{\alpha}} A_{\|}\right)+\left[\Gamma_{1_{\alpha}} \varphi, \frac{m_{\alpha}}{m_{i}} U_{\alpha}+\operatorname{sgn}\left(q_{\alpha}\right) \Gamma_{1_{\alpha}} A_{\|}\right]+\operatorname{sgn}\left(q_{\alpha}\right)\left[\Gamma_{2_{\alpha}} \varphi, \Gamma_{2_{\alpha}} A_{\|}\right] \\
& +\frac{m_{\alpha}}{m_{i}}\left[\Gamma_{2_{\alpha}} \varphi, Q_{\perp_{\alpha}}\right]-\frac{T_{\alpha}}{T_{e}}\left[\Gamma_{1_{\alpha}} A_{\|}, N_{\alpha}\right]-\frac{T_{\alpha}}{T_{e}}\left[\Gamma_{1_{\alpha}} A_{\|}, T_{\|_{\alpha}}\right]-\frac{T_{\alpha}}{T_{e}}\left[\Gamma_{2_{\alpha}} A_{\|}, T_{\perp_{\alpha}}\right] \\
& +\frac{\partial}{\partial z}\left(\operatorname{sgn}\left(q_{\alpha}\right) \Gamma_{1_{\alpha}} \varphi+\frac{T_{\alpha}}{T_{e}} N_{\alpha}+\frac{T_{\alpha}}{T_{e}} T_{\|_{\alpha}}\right)=0 \\
& \frac{\partial T_{\|_{\alpha}}}{\partial t}+\left[\Gamma_{1_{\alpha}} \varphi, T_{\|_{\alpha}}\right]-2\left[\Gamma_{1_{\alpha}} A_{\|}, U_{\alpha}\right]-\left[\Gamma_{1_{\alpha}} A_{\|}, Q_{\|_{\alpha}}\right]-2\left[\Gamma_{2_{\alpha}} A_{\|}, Q_{\perp_{\alpha}}\right]+\frac{\partial}{\partial z}\left(2 U_{\alpha}+Q_{\|_{\alpha}}\right)=0
\end{aligned}
$$




$$
\begin{aligned}
& \frac{\partial T_{\perp_{\alpha}}}{\partial t}+\left[\Gamma_{1_{\alpha}} \varphi, T_{\perp_{\alpha}}\right]+\left[\Gamma_{2_{\alpha}} \varphi, N_{\alpha}\right]+2\left[\Gamma_{2_{\alpha}} \varphi, T_{\perp_{\alpha}}\right]-\left[\Gamma_{1_{\alpha}} A_{\|}, Q_{\perp_{\alpha}}\right]-\left[\Gamma_{2_{\alpha}} A_{\|}, U_{\alpha}\right] \\
& -2\left[\Gamma_{2_{\alpha}} A_{\|}, Q_{\perp_{\alpha}}\right]+\frac{\partial Q_{\perp_{\alpha}}}{\partial z}=0,
\end{aligned}
$$

completed by the quasi-neutrality relation

$$
\sum_{\alpha}\left(\operatorname{sgn}\left(q_{\alpha}\right)\left(\Gamma_{1_{\alpha}} N_{\alpha}+\Gamma_{2_{\alpha}} T_{\perp_{\alpha}}\right)+\frac{T_{e}}{T_{\alpha}}\left(\Gamma_{0_{\alpha}}-1\right) \varphi\right)=0,
$$

and by the parallel Ampère's law

$$
\nabla_{\perp}^{2} A_{\|}=-\frac{\beta_{e}}{2} \sum_{\alpha} \operatorname{sgn}\left(q_{\alpha}\right)\left(\Gamma_{1_{\alpha}} U_{\alpha}+\Gamma_{2_{\alpha}} Q_{\perp_{\alpha}}\right) .
$$

The above system is formulated on a domain $\mathcal{D}=\left\{(x, y, z):-L_{x} \leqslant x \leqslant L_{x},-L_{y} \leqslant y \leqslant\right.$ $\left.L_{y},-L_{z} \leqslant z \leqslant L_{z}\right\}$ with $L_{x}, L_{y}, L_{z}$ corresponding to normalized lengths. We assume the presence of a strong and uniform component of the magnetic field along $\hat{\mathbf{z}}$. The transverse component of the magnetic field is indicated with $\nabla A_{\|} \times \hat{\mathbf{z}}$, with $A_{\|}$denoting the fluctuations of the magnetic flux function, which are assumed to be small compared to the amplitude of the guide field. Fluctuations of the magnetic field along $\hat{\mathbf{z}}$, moreover, are neglected. The symbol $\nabla_{\perp}^{2} \equiv \partial_{x x}+\partial_{y y}$, also, indicates the perpendicular Laplacian operator, where derivatives are taken only with respect to coordinates describing the plane perpendicular to the guide field. Electrostatic potential fluctuations are indicated with $\varphi$. The index $\alpha$ denotes the particle species, with $\alpha=e$ and $\alpha=i$ referring to electrons and ions, respectively. The fields $N_{\alpha}, U_{\alpha}, T_{\|_{\alpha}}, T_{\perp_{\alpha}}, Q_{\|_{\alpha}}, Q_{\perp_{\alpha}}$ are all defined on $\mathcal{D}$ and denote the fluctuations of the gyrocenter density, fluid velocity along the magnetic field, parallel and perpendicular temperature, parallel and perpendicular heat flux, respectively. Note that, with respect to Scott (2010), we adopted a different definition for the parallel heat flux fluctuations $Q_{\|_{\alpha}}$ which corresponds to the heat flux fluctuations of Scott (2010) multiplied by a factor 2. The constants $q_{\alpha}, m_{\alpha}$ and $T_{\alpha}$, on the other hand, indicate the charge, the mass of the particle and the equilibrium temperature of the species $\alpha$, respectively. We indicated with $\Gamma_{0_{\alpha}}, \Gamma_{1_{\alpha}}$ and $\Gamma_{2_{\alpha}}$ the gyroaverage operators, relative to the species $\alpha$, which commonly appear in gyrofluid models (Brizard 1992; Dorland \& Hammett 1993; Hammett et al. 1993; Beer \& Hammett 1996; Scott 2010). In Fourier space, assuming periodic boundary conditions along the $y$ and $z$ directions, these are defined, in terms of dimensional quantities, as

$$
\begin{aligned}
\Gamma_{0_{\alpha}} & =I_{0}\left(b_{\alpha}\right) \exp \left(-b_{\alpha}\right), \\
\Gamma_{1_{\alpha}} & =\frac{1}{n_{0}} \int d \mathcal{W}_{\alpha} \mathcal{F}_{e q_{\alpha}} J_{0}\left(\hat{k}_{\perp} \sqrt{2 \mu_{\alpha} m_{\alpha} c^{2} /\left(\left|q_{\alpha}\right|^{2} B_{0}\right)}\right), \\
\Gamma_{2_{\alpha}} & =\frac{1}{n_{0}} \int d \mathcal{W}_{\alpha}\left(\frac{\mu_{\alpha} B_{0}}{T_{\alpha}}-1\right) \mathcal{F}_{e q_{\alpha}} J_{0}\left(\hat{k}_{\perp} \sqrt{2 \mu_{\alpha} m_{\alpha} c^{2} /\left(\left|q_{\alpha}\right|^{2} B_{0}\right)}\right),
\end{aligned}
$$

where $b_{\alpha}=\hat{k}_{\perp}^{2} \rho_{\alpha}^{2}$, with $\hat{k}_{\perp}=\left(\hat{k}_{x}^{2}+\hat{k}_{y}^{2}\right)^{1 / 2}$ corresponding to the dimensional wavenumber in the direction perpendicular to the guide field. The wavenumbers $\hat{k}_{x}$ and $\hat{k}_{y}$ are defined as $\hat{k}_{x}=2 \pi m /\left(2 \hat{L}_{x}\right)$ and $\hat{k}_{y}=2 \pi n /\left(2 \hat{L}_{y}\right)$, respectively, with $m \in \mathbb{Z}, n \in \mathbb{Z}$ and with $\hat{L}_{x}=\rho_{s} L_{x}, \hat{L}_{y}=\rho_{s} L_{y}$ corresponding to the lengths $L_{x}$ and $L_{y}$ without normalization. The sonic Larmor radius $\rho_{s}$ is defined in Eq. (2.13). The symbol $\rho_{\alpha}$ is defined by $\rho_{\alpha}=$ $\left(T_{\alpha} / m_{\alpha}\right)^{1 / 2} /\left(\left|q_{\alpha}\right| B_{0} /\left(m_{\alpha} c\right)\right)$ and corresponds to the thermal gyroradius characteristic of the species $\alpha$, with $c$ equal to the speed of light. In Eqs. (2.7)-(2.9), $I_{0}$ and $J_{0}$ indicate the zeroth order modified Bessel function and Bessel function of the first kind, respectively. 
We also denoted by $n_{0}$ a uniform equilibrium gyrocenter density, with $B_{0}$ the amplitude of the magnetic guide field, with $\mu_{\alpha}$ the magnetic moment for particles of species $\alpha$ and with $d \mathcal{W}_{\alpha}=\left(2 \pi B_{0} / m_{\alpha}\right) d \mu_{\alpha} d v$ the volume element in velocity space, with $v$ indicating the velocity coordinate along the magnetic guide field. The physical meaning of the constant parameter $\beta_{e}$ was already anticipated in Sec. 1 and its explicit expression is given by $\beta_{e}=8 \pi n_{0} T_{e} / B_{0}^{2}$. As is common in the so-called $\delta f$ approach, the gyrocenter distribution functions are assumed to be close to equilibrium Maxwellian functions $\mathcal{F}_{e q_{\alpha}}$ defined as

$$
\mathcal{F}_{e q_{\alpha}}\left(v, \mu_{\alpha}\right)=n_{0}\left(\frac{m_{\alpha}}{2 \pi T_{\alpha}}\right)^{3 / 2} \exp \left(-\frac{m_{\alpha} v^{2}}{2 T_{\alpha}}-\frac{\mu_{\alpha} B_{0}}{T_{\alpha}}\right) .
$$

As indicated in Dorland \& Hammett (1993), Beer \& Hammett (1996) and Scott (2010), approximated expressions for the gyroaverage operators $\Gamma_{1_{\alpha}}$ and $\Gamma_{2_{\alpha}}$ are given by

$$
\Gamma_{1_{\alpha}}=\Gamma_{0_{\alpha}}^{1 / 2}, \quad \Gamma_{2_{\alpha}}=b_{\alpha} \frac{\partial \Gamma_{1_{\alpha}}}{\partial b_{\alpha}} .
$$

The system (2.1)-(2.4) is formulated making use of normalized variables, which are defined by

$$
\begin{aligned}
& t=\Omega_{i} \hat{t}, \quad x=\frac{\hat{x}}{\rho_{s}}, \quad y=\frac{\hat{y}}{\rho_{s}}, \quad z=\frac{\hat{z}}{\rho_{s}}, \\
& N_{\alpha}=\frac{\hat{N}_{\alpha}}{n_{0}}, \quad U_{\alpha}=\frac{\hat{U}_{\alpha}}{c_{s}}, \quad T_{\|_{\alpha}}=\frac{\hat{T}_{\|_{\alpha}}}{n_{0} T_{\alpha}}, \quad T_{\perp_{\alpha}}=\frac{\hat{T}_{\perp_{\alpha}}}{n_{0} T_{\alpha}}, \\
& Q_{\|_{\alpha}}=\frac{\hat{Q}_{\|_{\alpha}}}{n_{0} T_{\alpha} c_{s}}, \quad Q_{\perp_{\alpha}}=\frac{\hat{Q}_{\perp_{\alpha}}}{n_{0} T_{\alpha} c_{s}}, \\
& \varphi=\frac{e \hat{\varphi}}{T_{e}}, \quad A_{\|}=\frac{\hat{A}_{\|}}{B_{0} \rho_{s}} .
\end{aligned}
$$

In Eq. (2.12) we introduced the proton charge $e=\left|q_{e}\right|$, we denoted with a caret the dimensional quantities and we introduced the following constants:

$$
\Omega_{i}=\frac{e B_{0}}{m_{i} c}, \quad c_{s}=\sqrt{\frac{T_{e}}{m_{i}}}, \quad \rho_{s}=\frac{c_{s}}{\Omega_{i}},
$$

which correspond to the ion cyclotron frequency (referred to the guide field), the sound speed and the sonic Larmor radius, respectively.

In order to make contact also with the underlying $\delta f$ gyrokinetic theory, we specify that the dimensional moment fluctuations are defined as

$$
\begin{aligned}
& \hat{N}_{\alpha}=\int d \mathcal{W}_{\alpha} \hat{f}_{\alpha}, \quad \hat{U}_{\alpha}=\frac{1}{n_{0}} \int d \mathcal{W}_{\alpha} v \hat{f}_{\alpha} \\
& \hat{T}_{\|_{\alpha}}=\frac{1}{n_{0}} \int d \mathcal{W}_{\alpha}\left(m_{\alpha} v^{2}-T_{\alpha}\right) \hat{f}_{\alpha}, \quad \hat{T}_{\perp_{\alpha}}=\frac{1}{n_{0}} \int d \mathcal{W}_{\alpha}\left(\mu_{\alpha} B_{0}-T_{\alpha}\right) \hat{f}_{\alpha} \\
& \hat{Q}_{\|_{\alpha}}=\int d \mathcal{W}_{\alpha}\left(m_{\alpha} v^{2}-3 T_{\alpha}\right) v \hat{f}_{\alpha}, \quad \hat{Q}_{\perp_{\alpha}}=\int d \mathcal{W}_{\alpha}\left(\mu_{\alpha} B_{0}-T_{\alpha}\right) v \hat{f}_{\alpha}
\end{aligned}
$$

where $\hat{f}_{\alpha}=\hat{f}_{\alpha}\left(x, y, z, v, \mu_{\alpha}, t\right)$ is the perturbation of the gyrocenter distribution function related to the species $\alpha$.

The parent model (2.1)-(2.6) can be seen as a simplified version of the gyrofluid model (although written with a different normalization) of Scott (2010), taken in the non-dissipative limit and neglecting background inhomogeneities as well as evolution equations for the heat fluxes. The system (2.1)-(2.6), in particular, is evidently not closed, 
for closure relations for the heat flux fluctuations were not provided at this stage. Whereas the model of Scott (2010) includes evolution equations for the heat flux fluctuations, the system (2.1)-(2.6) does not. In order to obtain a simple model although retaining the effects of Landau damping, we decided to truncate the gyrofluid hierarchy at the lowest level where it is of interest to introduce Landau closures, that is at the level of temperature evolution equations.

As above anticipated, the choice of a simplified version of the model of Scott (2010) as parent model is motivated by the fact that its gyrofluid closures are determined in such a way that the model also conserves energy in the absence of heat flux fluctuations. Also, the parent model retains electron inertia, which we require for driving magnetic reconnection. In order to facilitate the comparison with Scott (2010) we specify that the parent model consisting of Eqs. (2.1)-(2.4), (2.5) and (2.6) can be obtained from Eqs. (163)-(166), (132) and (133) of Scott (2010) (where dissipation and background inhomogeneities are already omitted and when also the two-dimensional approximation for the fluctuations is assumed) by making the following formal replacements

$$
\begin{aligned}
& n_{z} \rightarrow N_{\alpha}, \quad u_{z \|} \rightarrow U_{\alpha}, \quad T_{z \|} \rightarrow T_{\|_{\alpha}}, \\
& T_{z \perp} \rightarrow T_{\perp_{\alpha}}, \quad q_{z \|} \rightarrow(1 / 2) Q_{\|_{\alpha}}, \quad q_{z \perp} \rightarrow Q_{\perp_{\alpha}}, \\
& A_{\|} \rightarrow A_{\|} / \beta_{e}, \quad \phi \rightarrow \varphi, \quad \beta_{e} A_{G} \rightarrow \Gamma_{1_{\alpha}} A_{\|}, \\
& \phi_{G} \rightarrow \Gamma_{1_{\alpha}} \varphi, \quad \beta_{e} \chi_{G} \rightarrow \Gamma_{2_{\alpha}} A_{\|}, \quad \Omega_{G} \rightarrow \Gamma_{2_{\alpha}} \varphi, \\
& p_{z \|} \rightarrow N_{\alpha}+T_{\|_{\alpha}}, \quad \beta_{e} \rightarrow \beta_{e} / 2, \quad \mu_{z} \rightarrow \operatorname{sgn}\left(q_{\alpha}\right) m_{\alpha} / m_{i}, \\
& \tau_{z} \rightarrow \operatorname{sgn}\left(q_{\alpha}\right) T_{\alpha} / T_{e}, \quad a_{z} \rightarrow \operatorname{sgn}\left(q_{\alpha}\right), \quad \Gamma_{i} \rightarrow \Gamma_{i_{\alpha}} \quad \text { for } i=0,1,2 .
\end{aligned}
$$

Also, because Eqs. (163)-(166), (132) and (133) of Scott (2010) refer to a two-dimensional limit, in order to retrieve Eqs. (2.1)-(2.4) of the present paper one needs to add terms accounting for the dependence on the $z$ coordinate. This is accomplished by replacing the operator $\beta_{e}\left[A_{G},.\right]$ with the operator $\left[\Gamma_{1_{\alpha}} A_{\|},.\right]-\partial_{z}$.

\subsection{Derivation of the model}

We consider a plasma consisting of electrons and of one single ionized ion species. The derivation of the reduced Landau-gyrofluid model proceeds essentially by an asymptotic expansion based on a prescribed scaling, and on imposing Landau-fluid closures on the heat fluxes. The asymptotic expansion is based on two small, independent parameters $\delta$ and $\epsilon$ defined by

$$
\delta^{2}=\frac{m_{e}}{m_{i}}, \quad \epsilon=\frac{1}{\Omega_{i} \mathcal{T}},
$$

with $\mathcal{T}$ indicating the characteristic time scale of variation of the fluctuating fields $N_{e, i}$, $U_{e, i}, T_{\|_{e, i}}$ and $T_{\perp_{e, i}}$ (which is assumed to be the same for all such fields). We also introduce the parameter

$$
\tau=\frac{T_{i}}{T_{e}},
$$

expressing the equilibrium temperature ratio.

The following ordering is assumed:

$$
\begin{aligned}
& \delta^{2} \ll 1, \quad \tau \sim k_{\perp}=\mathcal{O}(1), \\
& U_{e} \sim Q_{\|_{e}} \sim Q_{\perp_{e}}=\mathcal{O}\left(\frac{\epsilon}{\delta}\right), \\
& \frac{\partial}{\partial t} \sim N_{e} \sim T_{\|_{e}} \sim T_{\perp_{e}} \sim N_{i} \sim T_{\|_{i}} \sim T_{\perp_{i}} \sim \varphi=\mathcal{O}(\epsilon) \ll 1,
\end{aligned}
$$




$$
\begin{aligned}
& k_{z} \sim U_{i} \sim Q_{\|_{i}} \sim Q_{\perp_{i}} \sim A_{\|}=\mathcal{O}(\delta \epsilon), \\
& \beta_{e}=\mathcal{O}\left(\delta^{2}\right) .
\end{aligned}
$$

Assuming $\delta^{2} \ll 1$ implies $b_{e} \ll 1$, and consequently $\Gamma_{0 e} \simeq 1, \Gamma_{1 e} \simeq 1, \Gamma_{2 e} \simeq 0$, which allows us to neglect finite electron Larmor radius effects due to the gyroaverage operators. On the other hand, the ordering on $A_{\|}, \beta_{e}, k_{\perp}$ and $U_{e}$ retains finite electron inertia in the electron momentum equation, thus allowing for reconnection, and balances parallel current density with the parallel electron velocity in Ampère's law. In particular, as anticipated in Sec. 1, together with the requirement of neglecting electron Larmor radius effects, this leads to $\beta_{e}=\mathcal{O}\left(\delta^{2}\right) \ll 1$ (Zocco \& Schekochihin 2011). The ordering on $N_{e, i}, k_{\perp}, \varphi$, and $\omega$ reflects the ordering of the original parent model. In particular, it is consistent with the requirement that the $\mathbf{E} \times \mathbf{B}$ drift be the dominant drift on the characteristic time scale $\left(\partial_{t} \sim[\varphi,].\right)$ and that small amplitude electron and ion gyrocenter density fluctuations be of the same order as polarization terms at the characteristic perpendicular scale $\left(N_{i} \sim N_{e} \sim\left(\Gamma_{0 i}-1\right)(\varphi / \tau)\right)$ in the quasi-neutrality relation. We also remark that, as a consequence of the ordering, the dimensional electromagnetic potentials satisfy $\hat{\varphi} \sim\left(v_{\text {the }} / c\right) \hat{A}_{\|}$, with $v_{\text {the }}$ indicating the electron thermal speed. The ordering on $k_{z}$ comes from the strong anisotropy assumption $\left(k_{z} \ll k_{\perp}\right)$ and also from the requirement that the two contributions to the gradient operator along the magnetic field, be of the same order $\left(\left[A_{\|},.\right] \sim k_{z}\right)$. Investigations of magnetic reconnection (Comisso et al. 2012) performed adopting a model (Waelbroeck \& Tassi 2012) compatible with such ordering, on the other hand, indicated that, if $U_{i}=0$ at $t=0$, then the role of the parallel ion gyrocenter velocity is marginal on the reconnection dynamics. Based on this result, we ordered $U_{i} \ll U_{e}$. In the present model we allow for parallel and perpendicular temperature fluctuations. Because we are primarily interested in the effect of Landau closures on the electron fluid, we order the heat fluxes in such a way that they enter the evolution equations for the electron temperatures associated with leading order terms. We allow for finite ion temperature effects in the model, thus we set $\tau \sim 1$, although the cold-ion limit will be considered in the simulations.

Applying the above ordering to Eqs. (2.1)-(2.6) and retaining only the lowest order terms in each equation yields the following reduced system

$$
\begin{aligned}
& \frac{\partial N_{e}}{\partial t}+\left[\varphi, N_{e}\right]+\nabla_{\|} U_{e}=0, \\
& \frac{\partial\left(A_{\|}-\delta^{2} U_{e}\right)}{\partial t}+\left[\varphi, A_{\|}-\delta^{2} U_{e}\right]-\nabla_{\|}\left(N_{e}+T_{\|_{e}}\right)+\frac{\partial \varphi}{\partial z}=0, \\
& \frac{\partial T_{\|_{e}}}{\partial t}+\left[\varphi, T_{\|_{e}}\right]+\nabla_{\|}\left(2 U_{e}+Q_{\|_{e}}\right)=0, \\
& \frac{\partial T_{\perp_{e}}}{\partial t}+\left[\varphi, T_{\perp_{e}}\right]+\nabla_{\|} Q_{\perp_{e}}=0, \\
& \frac{\partial N_{i}}{\partial t}+\left[\Gamma_{1_{i}} \varphi, N_{i}\right]+\left[\Gamma_{2_{i}} \varphi, T_{\perp_{i}}\right]=0, \\
& \frac{\partial T_{\perp_{i}}}{\partial t}+\left[\Gamma_{1_{i}} \varphi, T_{\perp_{i}}\right]+2\left[\Gamma_{2_{i}} \varphi, T_{\perp_{i}}\right]+\left[\Gamma_{2_{i}} \varphi, N_{i}\right]=0, \\
& N_{e}-\Gamma_{1_{i}} N_{i}-\frac{\Gamma_{0_{i}}-1}{\tau} \varphi-\Gamma_{2_{i}} T_{\perp_{i}}=0, \\
& \nabla_{\perp}^{2} A_{\|}=\frac{\beta_{e}}{2} U_{e}
\end{aligned}
$$


where we introduced the parallel gradient operator

$$
\nabla_{\|} f=-\left[A_{\|}, f\right]+\frac{\partial f}{\partial z},
$$

for a function $f$. Equations (2.30)-(2.33) correspond to the evolution equations for the electron density, parallel momentum, parallel and perpendicular temperature, respectively. Equations (2.34) and (2.35) account for the evolution of ion gyrocenter density and perpendicular temperature, whereas Eqs. (2.36) and (2.37) are the quasi-neutrality relation and parallel Ampère's law.

It can be noticed that the system does not contain the evolution equations for the parallel ion gyrocenter velocity and for the parallel temperature. As a consequence of the ordering, such equations turn out to be decoupled from the system and thus we disregard them. Should one be interested in determing $U_{i}$ and $T_{\|_{i}}$, the required equations read

$$
\begin{aligned}
& \frac{\partial}{\partial t}\left(U_{i}+\Gamma_{1_{i}} A_{\|}\right)+\left[\Gamma_{1_{i}} \varphi, U_{i}+\Gamma_{1_{i}} A_{\|}\right]+\left[\Gamma_{2_{i}} \varphi, \Gamma_{2_{i}} A_{\|}\right]+\left[\Gamma_{2_{i}} \varphi, Q_{\perp_{i}}\right]-\tau\left[\Gamma_{1_{i}} A_{\|}, N_{i}\right] \\
& -\tau\left[\Gamma_{1_{i}} A_{\|}, T_{\|_{i}}\right]-\tau\left[\Gamma_{2_{i}} A_{\|}, T_{\perp_{i}}\right]+\frac{\partial}{\partial z}\left(\Gamma_{1_{i}} \varphi+\tau N_{i}+\tau T_{\|_{i}}\right)=0 \\
& \frac{\partial T_{\|_{i}}}{\partial t}+\left[\Gamma_{1_{i}} \varphi, T_{\|_{i}}\right]=0
\end{aligned}
$$

where a closure on $Q_{\perp_{i}}$ has to be determined.

We restrict, however, to the system (2.30)-(2.37) which we close by imposing the Landau fluid closures (Hammett \& Perkins 1990; Hammett et al. 1992; Snyder et al. 1997; Passot \& Sulem 2004; Sulem \& Passot 2015)

$$
Q_{\|_{e}}=-2 \alpha \mathcal{L} T_{\|_{e}}, \quad Q_{\perp_{e}}=-\alpha \mathcal{L} T_{\perp_{e}}
$$

where $\mathcal{L}$ holds for the Landau damping operator. Its modeling is discussed below. In Eq. (2.41), we furthermore introduced the constant $\alpha=(1 / \delta)(2 / \pi)^{1 / 2}$, originating from the linear kinetic theory. We point out that, as will be argued in Sec. 4.1, for the electron fluid with our ordering, temperature fluctuations and heat fluxes of the particles and of the gyrocenters coincide. Consequently, closures (2.41) keep the same form when expressed in terms of particle moments.

As mentioned in Snyder et al. (1997), a proper calculation of Landau damping would involve at each point of the computational domain and every time step, the evaluation of a convolution integral along the magnetic field line attached to this point, a task which exceeds the capability of the present day computers. A drastic simplification consists in linearizing the operator near a uniform ambient magnetic field, and thus replacing the local magnetic field lines by straight magnetic lines. The Landau damping operator then reduces to the negative of the Hilbert transform in the direction of the ambient magnetic field (defined by the unit vector $\mathbf{b}_{0}$ ) and, in this case, corresponds in Fourier space to the multiplicator $i \mathbf{b}_{0} \cdot \mathbf{k} /\left|\mathbf{b}_{0} \cdot \mathbf{k}\right|$. In one space dimension, this description is exact in the weakly nonlinear asymptotics (Ott \& Sudan 1969), and remains satisfactory in the fully nonlinear regime (Laveder et al. 2011). It however turns out to be inappropriate in several dimensions, as it may lead to spurious instabilities (Passot et al. 2014). In this case, indeed, the distortion of the magnetic field lines should be taken into account. Various modeling have been suggested for this purpose. Sharma et al. (2006) expressed the Landau damping operator in physical space in the form $\left(1 / k_{L}\right) \mathbf{b} \cdot \boldsymbol{\nabla}=\left(1 / k_{L}\right) \nabla_{\|}$ where $\mathbf{b}=\mathbf{B} /|\mathbf{B}|$ is the unit vector in the direction of the local magnetic field and $k_{L}$ a positive characteristic wavenumber associated with the scale of collisionless damping. This representation however does not preserve the zeroth order of the Landau operator 
and its nonlocality in physical space, which are replaced by a normalized first order derivative along the corresponding magnetic field line. This choice greatly alters the linear behavior (except at the chosen wavenumber $k_{L}$ ), effectively replacing Landau damping by a usual collisional dissipation. Differently, in Scott (1997), the degree of the Landau operator is preserved by reducing the operator to a multiplicative constant chosen to fit the large-scale dynamics. A Laplacian-type damping term was added in Scott (2002) and in subsequent publications by this author in order to provide a better matching at smaller scales. In this description, the nonlocality of the Landau operator is lost. With the aim of preserving the degree of the damping operator, while retaining its space nonlocality, a different modeling, first introduced in Passot et al. (2014), was used in Sulem et al. (2016) or Kobayashi et al. (2017). This is the modelling we will implement here. Indicating with $\check{f}_{\mathbf{k}}$ the $\mathbf{k}$-Fourier coefficient of $f$, where $\mathbf{k}=\left(k_{x}, k_{y}, k_{z}\right)$, the Landau damping operator is phenomenologically modeled in Fourier space as

$$
\check{\mathcal{L} f_{\mathbf{k}}}=\left[(\mathbf{k} \cdot \bar{\tau} \cdot \mathbf{k})^{-1 / 2}\right] \nabla_{\|} f_{\mathbf{k}}
$$

where $\bar{\tau}=\langle\mathbf{b} \otimes \mathbf{b}\rangle$ with \langle\rangle the spatial average over the domain $\mathcal{D}$. In particular, this form reduces to the correct formula in the linear case (i.e. when magnetic field lines are straight). We point out that, in particular for the application to the magnetic reconnection problem treated in Sec. 4, the field $A_{\|}$is further decomposed as $A_{\|}(x, y, z, t)=A_{\| e q}(x)+\epsilon \tilde{A}_{\|}(x, y, z, t)$, which has to be taken into account when expanding the operator $\mathcal{L}$ in terms of the small parameter $\epsilon$. Considering the ordering (2.25)-(2.29), one can then see that, according to the closures (2.41), the expansion performed on the operator $\mathcal{L}$ has to be of order $\mathcal{O}(1)$, so that $\nabla_{\|} Q_{\|_{e}} \sim \nabla_{\|} Q_{\perp_{e}} \sim \epsilon^{2}$, consistently with the order of the other terms present in Eqs. (2.32)-(2.33).

As a consequence of the ordering, the evolution of the ion gyrocenter moments $N_{i}$ and $T_{\perp_{i}}$ is essentialy electrostatic and neglects variations along the guide field direction. Moreover, choosing $N_{i}=T_{\perp_{i}}=0$ as initial conditions, one has $N_{i}=T_{\perp_{i}}=0$ as solutions. In this limit, the model yields a fluid reduction, with electron temperature anisotropy, of the hybrid kinetic-fluid model of Zocco \& Schekochihin (2011). Neglecting temperature fluctuations, one retrieves (up to the normalization) the two-field model derived in Schep et al. (1994) and investigated in Borgogno et al. (2005).

\section{Hamiltonian formulation of the model in the non-dissipative limit}

The Landau fluid closure (2.41) introduces dissipation in the system. We show that, in the non-dissipative limit $Q_{\|_{e}}=Q_{\perp_{e}}=0$, the model possesses a Hamiltonian structure, like previous reduced fluid models for inertial reconnection (Schep et al. 1994; Tassi et al. 2008; Waelbroeck et al. 2009; Waelbroeck \& Tassi 2012; Keramidas Charidakos et al. 2015; Grasso \& Tassi 2015).

As described for instance in Morrison (1998) and Marsden \& Ratiu (2002), showing that the dynamical system (2.30)-(2.35), complemented by the relations (2.36)-(2.37) and closed by $Q_{\|_{e}}=Q_{\perp_{e}}=0$, possesses a Hamiltonian structure, amounts to find a set of $N$ dynamical variables $\chi=\left(\chi_{1}, \cdots, \chi_{N}\right)$, a Hamiltonian functional $H=H\left(\chi_{1}, \cdots, \chi_{N}\right)$ and a Poisson bracket $\{$,$\} such that the evolution equations (2.30)-(2.35) can be cast in$ the form

$$
\frac{\partial \chi_{i}}{\partial t}=\left\{\chi_{i}, H\right\}, \quad i=1, \cdots, N
$$

We recall that the Poisson bracket is a bilinear antisymmetric operator which must satisfy the Leibniz and the Jacobi identity. Antisymmetry of the Poisson bracket, in particular, implies $\partial_{t} H=0$, reflecting the conservation of the Hamiltonian, i.e. of total energy of 
the system. We recall that the Leibniz identity reads $\{F G, K\}=F\{G, K\}+G\{F, K\}$, whereas the Jacobi identity corresponds to $\{\{F, G\}, K\}+\{\{G, K\}, F\}+\{\{K, F\}, G\}=0$, with $F, G$ and $K$ arbitrary functionals of the dynamical variables. For the model under consideration, $N=6$ and a suitable set of dynamical variables is given by the set of fields $\chi_{1}=n_{e}, \chi_{2}=A_{e} \equiv A_{\|}-\delta^{2} U_{e}, \chi_{3}=T_{\|_{e}}, \chi_{4}=T_{\perp_{e}}, \chi_{5}=N_{i}, \chi_{6}=T_{\perp_{i}}$. An effective procedure for constructing the Hamiltonian structure of the model is the one followed in the above mentioned Hamiltonian models for magnetic reconnection and consists in identifying first a conserved functional $H$ to consider as candidate Hamiltonian. Subsequently, a Poisson bracket is sought of the form

$$
\{F, G\}=\sum_{i, j, k=1}^{6} W_{k}^{i j} \int d^{3} x \chi_{k}\left[\frac{\delta F}{\delta \chi_{i}}, \frac{\delta G}{\delta \chi_{j}}\right]+\sum_{i, j=1}^{6} A^{i j} \frac{\delta F}{\delta \chi_{i}} \frac{\partial}{\partial z} \frac{\delta G}{\delta \chi_{j}},
$$

for two functionals $F$ and $G$. In Eq. (3.2) $W_{k}^{i j}$ and $A^{i j}$ are constant coefficients to be determined by imposing that $\{$,$\} satisfies antisymmetry and the Jacobi identity$ (bilinearity and the Leibniz identity are automatically satisfied by the form (3.2)) and also that, when combined with the candidate Hamiltonian $H$ by means of the expression (3.1), it yields the model equations.

For the model under consideration, it is not difficult to see that, assuming that boundary terms vanish when integrating by parts, the functional

$$
\begin{aligned}
& H=\frac{1}{2} \int d^{3} x\left(N_{e}^{2}+N_{i}^{2}-U_{e} A_{e}+\frac{T_{\|_{e}}^{2}}{2}+T_{\perp_{e}}^{2}+T_{\perp_{i}}^{2}-\varphi \frac{\Gamma_{0_{i}}-1}{\tau} \varphi\right) \\
& =\frac{1}{2} \int d^{3} x\left(N_{e}^{2}+N_{i}^{2}+\delta^{2} U_{e}^{2}+\frac{2}{\beta_{e}}\left|\nabla_{\perp} A_{\|}\right|^{2}+\frac{T_{\|_{e}}^{2}}{2}+T_{\perp_{e}}^{2}+T_{\perp_{i}}^{2}\right. \\
& \left.-N_{e} \varphi+N_{i} \Gamma_{1_{i}} \varphi+T_{\perp_{i}} \Gamma_{2_{i}} \varphi\right)
\end{aligned}
$$

is conserved by Eqs. (2.30)-(2.35). The formulation (3.3) makes it easy to see that the Hamiltonian functional $H$ is given by the sum of internal energy given by electron and ion density fluctuations (first two terms of Eq. (3.3)), parallel kinetic energy and magnetic energy (third and fourth term of Eq. (3.3)), internal energy due to electron and ion temperature fluctuations (fifth, sixth and seventh term of Eq. (3.3)) and electrostatic energy due to polarization effects (last three terms of Eq. (3.3)). Note that, in spite of the small parameters $\delta^{2}$ and $\beta_{e}$ present in the expression (3.3), all terms contributing to the Hamiltonian are of the same order, according to the ordering (2.25)-(2.29).

With regard to the Poisson bracket, it can be shown that it is given by

$$
\{F, G\}=\{F, G\}_{e}-\{F, G\}_{i},
$$

where

$$
\begin{aligned}
& \{F, G\}_{e}=\int d^{3} x\left(N_{e}\left(\left[F_{N_{e}}, G_{N_{e}}\right]+\delta^{2}\left[F_{A_{e}}, G_{A_{e}}\right]+2\left[F_{{\|_{\|}}_{e}}, G_{T_{\|_{e}}}\right]+\left[F_{T_{\perp_{e}}}, G_{T_{\perp_{e}}}\right]\right)\right. \\
& +A_{e}\left(\left[F_{A_{e}}, G_{N_{e}}\right]+\left[F_{N_{e}}, G_{A_{e}}\right]+2\left(\left[F_{A_{e}}, G_{T_{\|_{e}}}\right]+\left[F_{T_{\|_{e}}}, G_{A_{e}}\right]\right)\right) \\
& +T_{\|_{e}}\left(\delta^{2}\left[F_{A_{e}}, G_{A_{e}}\right]+\left[F_{N_{e}}, G_{T_{\|_{e}}}\right]+\left[F_{T_{\|_{e}}}, G_{N_{e}}\right]+\left[F_{T_{\|_{e}}}, G_{T_{\|_{e}}}\right]-\frac{1}{2}\left[F_{T_{\perp_{e}}}, G_{T_{\perp_{e}}}\right]\right) \\
& +T_{\perp_{e}}\left(\left[F_{N_{e}}, G_{T_{\perp_{e}}}\right]+\left[F_{T_{\perp_{e}}}, G_{N_{e}}\right]-\left[F_{T_{\perp_{e}}}, G_{T_{\|_{e}}}\right]-\left[F_{T_{\|_{e}}}, G_{T_{\perp_{e}}}\right]+h\left[F_{T_{\perp_{e}}}, G_{T_{\perp_{e}}}\right]\right) \\
& \left.+F_{N_{e}} \frac{\partial}{\partial z} G_{A_{e}}+F_{A_{e}} \frac{\partial}{\partial z} G_{N_{e}}+2 F_{T_{\|_{e}}} \frac{\partial}{\partial z} G_{A_{e}}+2 F_{A_{e}} \frac{\partial}{\partial z} G_{T_{\|_{e}}}\right)
\end{aligned}
$$


and

$$
\begin{aligned}
& \{F, G\}_{i}=\int d^{3} x\left(N_{i}\left(\left[F_{N_{i}}, G_{N_{i}}\right]+\left[F_{T_{\perp_{i}}}, G_{T_{\perp_{i}}}\right]\right)\right. \\
& \left.+T_{\perp_{i}}\left(\left[F_{N_{i}}, G_{T_{\perp_{i}}}\right]+\left[F_{T_{\perp_{i}}}, G_{N_{i}}\right]+2\left[F_{T_{\perp_{i}}}, G_{T_{\perp_{i}}}\right]\right)\right) .
\end{aligned}
$$

In Eqs. (3.5)-(3.6), the subscripts on functionals indicate functional derivatives, so that, for instance, $F_{A_{e}}=\delta F / \delta A_{e}$, whereas $h$ is an arbitrary constant, so that, actually, expression (3.4) can be viewed as a one-parameter family of Poisson brackets. This Poisson bracket is of the prescribed form (3.2) but the formulation (3.4) makes it easy to see that it can be written as the direct sum of two Poisson brackets $\{,\}_{e}$ and $\{,\}_{i}$ which depend only on functionals pertaining to the electrons and ion gyrocenters, respectively. As in previous Hamiltonian models (Waelbroeck et al. 2009; Waelbroeck \& Tassi 2012; Grasso \& Tassi 2015; Keramidas Charidakos et al. 2015; Tassi 2015, 2016), in this formulation, the coupling between electron and gyrocenter ion dynamics is provided by the Hamiltonian. Notice also that, in spite of the small parameter $\delta^{2}$ multiplying two terms in the Poisson bracket (3.5), all the resulting terms in the equations of motion end up to be of the same order. In the specific case of the two terms with coefficient $\delta^{2}$, this comes as a consequence of the fact that the functional derivative $H_{A_{e}}=-\left(2 / \beta_{e}\right) \nabla_{\perp}^{2} A_{\|}$ is of order $\epsilon / \delta$, and all the terms in Eq. (2.31) are of order $\delta \epsilon^{2}$.

It can be checked by direct calculations that the Hamiltonian (3.3) and the Poisson bracket (3.4) yield Eqs. (2.30)-(2.35), independently on the value of $h$. However, as is the typical case for Hamiltonian fluid models expressed in terms of Eulerian variables, the Poisson bracket is of noncanonical type (see, e.g., Morrison (1998)). Thus, in our procedure, in particular, the above mentioned four properties defining a Poisson bracket are not automatically satisfied and must be verified. It is in particular not obvious to show that the Poisson bracket (3.4) satisfies the Jacobi identity (antisymmetry, on the other hand, is rather straightforward to show, whereas bilinearity and the Leibniz identity, as above mentioned, are already implied by the form (3.2). One way to verify the Jacobi identity is based on the results of Thiffeault \& Morrison (2000) and Tassi et al. (2010). According to such procedure, one proceeds by first showing that the part of the Poisson bracket (3.2) accounting for the perpendicular dynamics (i.e. setting $A^{i j}=0$ for $i, j=$ $1, \cdots, N)$ is itself a Poisson bracket. This amounts to show that the $N$ matrices $W^{(j)}$, defined by

$$
\left[W^{(j)}\right]_{k}^{i}=W_{k}^{i j}, \quad j=1, \cdots, N
$$

pairwise commute. Subsequently, the Jacobi identity for the entire bracket (3.2) is proved by showing that the coefficients $W_{k}^{i j}$ and the coefficients $A^{i j}$ satisfy the relations

$$
A^{r s} W_{r}^{i j}=A^{r j} W_{r}^{s i}=A^{r i} W_{r}^{j s},
$$

where the sum over the repeated index $r$ is understood. 
In the case of bracket (3.4) the six matrices $W^{(1)}, \cdots, W^{(6)}$ are given by

$$
\begin{aligned}
& W^{(1)}=\left(\begin{array}{cccccc}
1 & 0 & 0 & 0 & 0 & 0 \\
0 & 1 & 0 & 0 & 0 & 0 \\
0 & 0 & 1 & 0 & 0 & 0 \\
0 & 0 & 0 & 1 & 0 & 0 \\
0 & 0 & 0 & 0 & 0 & 0 \\
0 & 0 & 0 & 0 & 0 & 0
\end{array}\right), \quad W^{(2)}=\left(\begin{array}{cccccc}
0 & \delta^{2} & 0 & 0 & 0 & 0 \\
1 & 0 & 2 & 0 & 0 & 0 \\
0 & \delta^{2} & 0 & 0 & 0 & 0 \\
0 & 0 & 0 & 0 & 0 & 0 \\
0 & 0 & 0 & 0 & 0 & 0 \\
0 & 0 & 0 & 0 & 0 & 0
\end{array}\right), \\
& W^{(3)}=\left(\begin{array}{cccccc}
0 & 0 & 2 & 0 & 0 & 0 \\
0 & 2 & 0 & 0 & 0 & 0 \\
1 & 0 & 1 & 0 & 0 & 0 \\
0 & 0 & 0 & -1 & 0 & 0 \\
0 & 0 & 0 & 0 & 0 & 0 \\
0 & 0 & 0 & 0 & 0 & 0
\end{array}\right), \quad W^{(4)}=\left(\begin{array}{cccccc}
0 & 0 & 0 & 1 & 0 & 0 \\
0 & 0 & 0 & 0 & 0 & 0 \\
0 & 0 & 0 & -1 / 2 & 0 & 0 \\
1 & 0 & -1 & h & 0 & 0 \\
0 & 0 & 0 & 0 & 0 & 0 \\
0 & 0 & 0 & 0 & 0 & 0
\end{array}\right), \\
& W^{(5)}=\left(\begin{array}{cccccc}
0 & 0 & 0 & 0 & 0 & 0 \\
0 & 0 & 0 & 0 & 0 & 0 \\
0 & 0 & 0 & 0 & 0 & 0 \\
0 & 0 & 0 & 0 & 0 & 0 \\
0 & 0 & 0 & 0 & -1 & 0 \\
0 & 0 & 0 & 0 & 0 & -1
\end{array}\right), \quad W^{(6)}=\left(\begin{array}{cccccc}
0 & 0 & 0 & 0 & 0 & 0 \\
0 & 0 & 0 & 0 & 0 & 0 \\
0 & 0 & 0 & 0 & 0 & 0 \\
0 & 0 & 0 & 0 & 0 & 0 \\
0 & 0 & 0 & 0 & 0 & -1 \\
0 & 0 & 0 & 0 & -1 & -2
\end{array}\right),
\end{aligned}
$$

whereas the coefficients $A^{i j}$ are $A^{i j}=0$ for all $i, j=1, \cdots, 6$ except for $A^{12}=A^{21}=1$ and $A^{23}=A^{32}=2$.

It can then easily be verified that all matrices $W^{(1)}, \cdots, W^{(6)}$ pairwise commute (in particular, for any value of $h$ ) and that the relations (3.8) are satisfied, which proves the Jacobi identity for the Poisson bracket (3.4).

One important feature of noncanonical Poisson brackets is that they possess Casimir invariants, i.e. functionals $C$ such that $\{C, F\}=0$ for any functional $F$ of the dynamical variables. Because $\partial_{t} C=\{C, H\}=0$ Casimir invariants are indeed conserved by the dynamics. Their existence then implies constraints on the dynamics, in addition to that of energy conservation.

For the Poisson bracket (3.4), it can be found, by solving $\{C, F\}=0$ for arbitrary $F$, that Casimir invariants correspond to

$$
\begin{aligned}
C_{1} & =\int d^{3} x \nu_{+}, \\
C_{2} & =\int d^{3} x \nu_{-}, \\
C_{3} & =\int d^{3} x \mathcal{S}_{+}\left(s+\lambda_{+} T_{\perp_{e}}\right), \\
C_{4} & =\int d^{3} x \mathcal{S}_{-}\left(s+\lambda_{-} T_{\perp_{e}}\right), \\
C_{5} & =\int d^{3} x \mathcal{M}_{+}\left(\mu_{+}\right), \\
C_{6} & =\int d^{3} x \mathcal{M}_{-}\left(\mu_{-}\right),
\end{aligned}
$$

where

$$
\nu_{ \pm}= \pm \frac{\delta}{\sqrt{3}}\left(N_{e}+T_{\|_{e}}\right)+A_{e}
$$




$$
\begin{aligned}
& s=N_{e}-\frac{T_{\|_{e}}}{2}, \\
& \mu_{ \pm}=N_{i}+(1 \pm \sqrt{2}) T_{\perp_{i}}, \\
& \lambda_{ \pm}=\frac{1}{2}\left(h \pm \sqrt{h^{2}+6}\right),
\end{aligned}
$$

and where $\mathcal{S}_{ \pm}$and $\mathcal{M}_{ \pm}$indicate arbitrary functions. Note that, in spite of the small parameter $\delta$ appearing in the definition of the field variables $\nu_{ \pm}$, all terms on the righthand side of Eq. (3.16) are of the same order. In the $2 D$ limit with translational invariance along $z$, the two Casimir invariants $C_{1}$ and $C_{2}$ extend to two infinite families of Casimir invariants $C_{1,2}=\int d^{3} x \mathcal{N}_{ \pm}\left(\nu_{ \pm}\right)$with $\mathcal{N}_{ \pm}$arbitrary functions. This feature is common in Hamiltonian reduced fluid models for plasmas and is also visible from the form of the Casimir invariants $C_{5}$ and $C_{6}$, pertaining to ion dynamics, for which derivatives along $z$ play no role in the dynamics and in the Poisson bracket. Indeed, also $C_{5}$ and $C_{6}$ consist of infinite families of invariants. More interesting, on the other hand, are the Casimir invariants $C_{3}$ and $C_{4}$. They consist of infinite families also in the pure $3 D$ case. Also, given the arbitrariness of $h$, the coefficients $\lambda_{ \pm}$appearing in the expressions for $C_{3}$ and $C_{4}$ can also take infinite values. Defining $s_{ \pm}=s+\lambda_{ \pm} T_{\perp_{e}}$, one can perform the change of variables $\left(N_{e}, A_{e}, T_{\|_{e}}, T_{\perp_{e}}, N_{i}, T_{\perp_{i}}\right) \rightarrow\left(\nu_{+}, \nu_{-}, s_{+}, s_{-}, \mu_{+}, \mu_{-}\right)$and, in terms of the new set of variables, the Poisson bracket (3.4) takes its simplest, normal form

$$
\{F, G\}=\{F, G\}_{e}-\{F, G\}_{i},
$$

with

$$
\begin{aligned}
& \{F, G\}_{e}=\int d^{3} x\left(2 \sqrt{3} \delta \nu_{+}\left[F_{\nu_{+}}, G_{\nu_{+}}\right]-2 \sqrt{3} \delta \nu_{-}\left[F_{\nu_{-}}, G_{\nu_{-}}\right]\right. \\
& +\frac{6+h^{2}+h \sqrt{h^{2}+6}}{2} s_{+}\left[F_{s_{+}}, G_{s_{+}}\right]+\frac{6+h^{2}-h \sqrt{h^{2}+6}}{2} s_{-}\left[F_{s_{-}}, G_{s_{-}}\right] \\
& \left.+F_{\nu_{+}} \frac{\partial}{\partial z} G_{\nu_{+}}-F_{\nu_{-}} \frac{\partial}{\partial z} G_{\nu_{-}}\right)
\end{aligned}
$$

and

$$
\{F, G\}=\int d^{3} x\left(2(2+\sqrt{2}) \mu_{+}\left[F_{\mu_{+}}, G_{\mu_{+}}\right]+(4-2 \sqrt{2}) \mu_{-}\left[F_{\mu_{-}}, G_{\mu_{-}}\right]\right) .
$$

The set of variables $\left(\nu_{+}, \nu_{-}, s_{+}, s_{-}, \mu_{+}, \mu_{-}\right)$suggested by the form of the Casimir invariants can indeed be referred to as normal fields (Waelbroeck et al. 2009). The normal fields are also useful variables for unveiling some structures of the evolution equations of the system. Indeed, the evolution equations (2.30)-(2.35), formulated in terms of the normal fields, read

$$
\begin{aligned}
& \frac{\partial \nu_{+}}{\partial t}+\left[\phi_{+_{e}}, \nu_{+}\right]+\frac{\partial}{\partial z}\left(\phi_{+_{e}}-\frac{\sqrt{3}}{\delta} \nu_{+}\right)=0, \\
& \frac{\partial \nu_{-}}{\partial t}+\left[\phi_{-_{e}}, \nu_{-}\right]+\frac{\partial}{\partial z}\left(\phi_{-_{e}}+\frac{\sqrt{3}}{\delta} \nu_{-}\right)=0, \\
& \frac{\partial s_{+}}{\partial t}+\left[\varphi, s_{+}\right]=0, \\
& \frac{\partial s_{-}}{\partial t}+\left[\varphi, s_{-}\right]=0, \\
& \frac{\partial \mu_{+}}{\partial t}+\left[\phi_{+_{i}}, \mu_{+}\right]=0
\end{aligned}
$$




$$
\frac{\partial \mu_{-}}{\partial t}+\left[\phi_{-i}, \mu_{-}\right]=0
$$

where

$$
\phi_{ \pm_{e}}=\varphi \pm \frac{\sqrt{3}}{\delta} A, \quad \phi_{ \pm_{i}}=\Gamma_{1_{i}} \varphi+(1 \pm \sqrt{2}) \Gamma_{2_{i}} \varphi
$$

act as generalized stream functions.

The reformulation in terms of normal fields makes it then possible to see that, in the $2 D$ limit, the system actually consists of advection equations for the normal fields, which can be seen as Lagrangian invariants advected by generalized stream functions. This feature is shared with other Hamiltonian models for reconnection (Waelbroeck et al. 2009; Waelbroeck \& Tassi 2012; Schep et al. 1994; Grasso \& Tassi 2015; Keramidas Charidakos et al. 2015). In particular, we remark that the variables $\nu_{ \pm}$are analogous to two normal fields found for the ion dynamics in the gyrofluid model of Keramidas Charidakos et al. (2015) and correspond to the diagonalizing variables introduced in Tassi (2015) in the context of Hamiltonian closures for drift-fluid models. A linear combination of Eqs. (3.27) and (3.28) yields

$$
\begin{aligned}
& \frac{\partial s}{\partial t}+[\varphi, s]=0, \\
& \frac{\partial T_{\perp_{e}}}{\partial t}+\left[\varphi, T_{\perp_{e}}\right]=0 .
\end{aligned}
$$

Equation (3.33) corresponds, evidently, to the original Eq. (2.33) with $Q_{\perp_{e}}=0$, expressing the advection of the perpendicular electron temperature fluctuations in the absence of heat flux. Equation (3.32), on the other hand, expresses the advection (already present in the model of Keramidas Charidakos et al. (2015), although referred to ion gyrofluid moments) of the field $s$ which, from its definition (3.17), measures the departure from a condition of adiabaticity with respect to the parallel temperature and can, in this sense, be seen as an expression of entropy conservation. Similarly, perpendicular electron temperature fluctuations, are purely advected by the $\mathbf{E} \times \mathbf{B}$ flow associated with the stream function $\varphi$. Due to finite ion gyroradius effects associated with the operator $\Gamma_{2_{i}}$, on the other hand, the ion gyrocenter perpendicular temperature fluctuations $T_{\perp_{i}}$, are not purely advected. Likewise, ion gyrocenter density fluctuations $N_{i}$, although obeying an electrostatic dynamics in our ordering, are not advected due to the finite ion gyroradius contribution associated with the last term in Eq. (2.34). The formulation (3.29)-(3.30) shows, however, that linear combinations of $N_{i}$ and $T_{\perp_{i}}$, corresponding to $\mu_{ \pm}$, get advected by the generalized velocity fields associated with the stream functions $\phi_{ \pm_{i}}$.

\section{Collisionless magnetic reconnection in the cold-ion regime}

As an application of the previously derived reduced Landau-gyrofluid model, we consider an analysis of the phenomenon of magnetic reconnection driven by electron inertia. The analysis is based on numerical simulations, and the relative simplicity of the reduced Landau-gyrofluid model makes it convenient to simulate reconnection also on a three-dimensional domain. Because we are interested mainly in the effect of Landau damping, we consider a further simplified version of the model which neglects ion temperature effects. In the following, we first present the cold-ion version of the model and subsequently describe results from the numerical simulations, concerning the linear growth rates and the nonlinear evolution. 


\subsection{Cold-ion limit}

We consider a simplified version of the model (2.30)-(2.37), where the ion response is determined only by the quasi-neutrality relation in the cold-ion limit. More specifically, we take the solutions $N_{i}=T_{\perp_{i}}=0$ for the ion gyrocenter density and perpendicular temperature fluctuations, and consider the limit $\tau \ll 1$. With these assumptions, the model reduces to

$$
\begin{aligned}
& \frac{\partial N_{e}}{\partial t}+\left[\varphi, N_{e}\right]+\nabla_{\|} U_{e}=0 \\
& \frac{\partial}{\partial t}\left(A_{\|}-\delta^{2} U_{e}\right)-\left[\varphi, \delta^{2} U_{e}\right]-\nabla_{\|}\left(N_{e}+T_{\|_{e}}-\varphi\right)=0 \\
& \frac{\partial T_{\|_{e}}}{\partial t}+\left[\varphi, T_{\|_{e}}\right]+\nabla_{\|}\left(Q_{\|_{e}}+2 U_{e}\right)=0 \\
& \frac{\partial T_{\perp_{e}}}{\partial t}+\left[\varphi, T_{\perp_{e}}\right]+\nabla_{\|} Q_{\perp_{e}}=0 \\
& \nabla_{\perp}^{2} \varphi=N_{e} \\
& \nabla_{\perp}^{2} A_{\|}=\frac{\beta_{e}}{2} U_{e} .
\end{aligned}
$$

In such cold-ion limit, it is also immediate to rewrite the model in terms of particle moments instead of gyrocenter moments. Actually, due to the assumptions $\beta_{e} \sim \delta^{2} \ll$ 1 , the electron gyrofluid moments are identical to the corresponding particle moments (Brizard 1992), i.e. $N_{e}=n_{e}, U_{e}=u_{e}, T_{\|_{e}}=t_{\|_{e}}, T_{\perp_{e}}=t_{\perp_{e}}, Q_{\|_{e}}=q_{\|_{e}}$ and $Q_{\perp_{e}}=q_{\perp_{e}}$ where $n_{e}, u_{e}, t_{\|_{e}}, t_{\perp_{e}}, q_{\|_{e}}$ and $q_{\perp_{e}}$ indicate the particle density, parallel velocity, parallel and perpendicular temperature and heat flux fluctuations, respectively, for the electron fluid. Ion dynamics enters only through the quasi-neutrality relation, which, in terms of particle moments, yields $n_{i}=\nabla_{\perp}^{2} \varphi=n_{e}$, where $n_{i}$ denotes the ion density fluctuations (which differ from the ion gyrocenter fluctuations $N_{i}$ ).

In terms of particle moments, the model (4.1)-(4.6) can then be reformulated as

$$
\begin{aligned}
& \frac{\partial \nabla_{\perp}^{2} \varphi}{\partial t}+\left[\varphi, \nabla_{\perp}^{2} \varphi\right]+\frac{2}{\beta_{e}} \nabla_{\|} \nabla_{\perp}^{2} A_{\|}=0 \\
& \frac{\partial}{\partial t}\left(A_{\|}-\frac{2}{\beta_{e}} \delta^{2} \nabla_{\perp}^{2} A_{\|}\right)-\frac{2}{\beta_{e}} \delta^{2}\left[\varphi, \nabla_{\perp}^{2} A_{\|}\right]-\nabla_{\|}\left(\nabla_{\perp}^{2} \varphi+t_{\|_{e}}-\varphi\right)=0 \\
& \frac{\partial t_{\|_{e}}}{\partial t}+\left[\varphi, t_{\|_{e}}\right]+\nabla_{\|}\left(q_{\|_{e}}+\frac{4}{\beta_{e}} \nabla_{\perp}^{2} A_{\|}\right)=0 \\
& \frac{\partial t_{\perp_{e}}}{\partial t}+\left[\varphi, t_{\perp_{e}}\right]+\nabla_{\|} q_{\perp_{e}}=0
\end{aligned}
$$

with

$$
q_{\|_{e}}=-2 \alpha \mathcal{L} t_{\|_{e}}, \quad q_{\perp_{e}}=-\alpha \mathcal{L} t_{\perp_{e}} .
$$

In the absence of temperature fluctuations (i.e. $t_{\|_{e}}=t_{\perp_{e}}=0$ ), the model corresponds, up to the normalization, to the two-field model investigated in Borgogno et al. (2005) and Grasso et al. (2007).

We remark that, when neglecting dissipation (i.e. $q_{\|_{e}}=q_{\perp_{e}}=0$ ), the above model still possesses a Hamiltonian structure. Indeed, the set of functionals of $N_{e}, A_{e}, T_{\|_{e}}$ and $T_{\perp_{e}}$ is a sub-algebra with respect to the Poisson bracket (3.4), and in particular with respect to the Poisson bracket $\{,\}_{e}$ of Eq. (3.5). The model (4.1)-(4.6), for $Q_{\|_{e}}=Q_{\perp_{e}}=0$ is then a Hamiltonian system with Poisson bracket given by $\{,\}_{e}$ and Hamiltonian functional 
given by

$$
H=\frac{1}{2} \int d^{3} x\left(N_{e}^{2}+\delta^{2} U_{e}^{2}+\frac{2}{\beta}\left|\nabla_{\perp} A_{\|}\right|^{2}+\frac{T_{\|_{e}}^{2}}{2}+T_{\perp_{e}}^{2}+\left|\nabla_{\perp} \varphi\right|^{2}\right) .
$$

The formulation of the Hamiltonian structure in terms of particle moments follows trivially because, as seen above, in this limit, electron gyrofluid moments identify with electron particle moments.

\subsection{Linear regime}

Equations (4.7)-(4.10) are integrated over the domain $\left\{-L_{x} \leqslant x \leqslant L_{x},-L_{y} \leqslant y \leqslant\right.$ $\left.L_{y},-L_{z} \leqslant z \leqslant L_{z}\right\}$. For the investigation of the linear growth rates, we chose $L_{x}=$ $46, L_{y}=24 \pi, L_{z}=96 \pi$. The initial condition is given by

$$
\begin{aligned}
& A_{\|}(x, y, z, 0)=-\lambda \ln \cosh \left(\frac{x}{\lambda}\right)+A_{0}\left(\frac{x-x_{s}}{\lambda}\right) \exp \left[i\left(k_{y} y+k_{z} z\right)\right]+c . c ., \\
& t_{\|_{e}}(x, y, z, 0)=0, \quad t_{\perp_{e}}(x, y, z, 0)=0, \quad \varphi(x, y, z, 0)=0,
\end{aligned}
$$

where $\lambda$ is a positive constant whose value, for these numerical simulations, has been set equal to 3 . The values of the parameters $\delta$ and $\beta_{e}$, on the other hand, have been set as $\delta=0.1$ and $\beta_{e}=0.05$, respectively. The initial condition (4.14)-(4.15) corresponds to perturbing a magnetic equilibrium $\mathbf{B}_{e q}(x)=\nabla A_{\| e q}(x) \times \hat{\mathbf{z}}+\hat{\mathbf{z}}=-\tanh (x / \lambda) \hat{\mathbf{y}}+\hat{\mathbf{z}}$ with a single-helicity disturbance, associated with two wave numbers $k_{y}=m \pi / L_{y}$ and $k_{z}=n \pi / L_{z}$, for non-negative integers $m$ and $n$, and where the helicity is determined by the ratio $k_{z} / k_{y}$. The amplitude of the perturbation is given by a function $A_{0}$ centered at the resonant surface $x=x_{s}$, the latter identified by the relation $-\tanh \left(x_{s} / \lambda\right)=k_{z} / k_{y}$. Because of the choice of the initial conditions, one can infer, from Eq. (4.10), that $t_{\perp_{e}}=0$ at all times, so that perpendicular temperature plays no role in these simulations.

For the numerical solution of Eqs. (4.7)-(4.10) we adopt further developments of the code described in Grasso et al. (2007). In particular the code has been parallelized along both the periodic directions $y$ and $z$ and the operator $\mathcal{L}$ of Eq. (2.41) has been introduced, exploiting its Fourier representation given in Eq. (2.42). The new code has been benchmarked in the non-dissipative isothermal limit against previous codes solving the equations described in Grasso et al. (2007) and Borgogno et al. (2005).

In the absence of parallel Landau damping $\left(q_{\|_{e}}=0\right)$, given the initial conditions, the parallel temperature follows an adiabatic behavior dictated by $t_{\|_{e}}=2 n_{e}=2 \nabla_{\perp}^{2} \varphi$. In this limit the model can be rewritten as

$$
\begin{aligned}
& \frac{\partial \nabla_{\perp}^{2} \varphi}{\partial t}+\left[\varphi, \nabla_{\perp}^{2} \varphi\right]+\frac{2}{\beta_{e}} \nabla_{\|} \nabla_{\perp}^{2} A_{\|}=0, \\
& \frac{\partial}{\partial t}\left(A_{\|}-\frac{2}{\beta_{e}} \delta^{2} \nabla_{\perp}^{2} A_{\|}\right)-\frac{2}{\beta_{e}} \delta^{2}\left[\varphi, \nabla_{\perp}^{2} A_{\|}\right]-\nabla_{\|}\left(3 \nabla_{\perp}^{2} \varphi-\varphi\right)=0 .
\end{aligned}
$$

For purely two-dimensional disturbances $(n=0)$, an analytical prediction of the linear growth rate of the perturbation for the system (4.16)-(4.17), with initial conditions for $A_{\|}$and $\varphi$ as given in Eqs. (4.14)-(4.15), can be obtained by properly adapting the result of Porcelli (1991) valid for isothermal electrons. Note, in fact, that the model equations in the adiabatic limit differ from those in the isothermal limit just by the factor 3 in the last but one term of Eq. (4.17), which becomes a factor 1 in the isothermal limit. This amounts to replace, in the expression for the growth rate of Porcelli (1991), the parameter $\hat{\rho}_{\tau}$ by $\sqrt{3} \hat{\rho}_{\tau}$. Assuming that in the linear regime the perturbations for $\varphi$ and 
$A_{\|}$are of the form $\bar{f}(x / \lambda) \exp \left[i\left(k_{y} y+k_{z} z\right)-\gamma t\right]+c . c .$, where $\bar{f}$ is a two-dimensional vector $\left(\bar{\varphi}, \overline{A_{\|}}\right)$, the expression for the growth rate $\gamma$ in the adiabatic limit then becomes

$$
\gamma_{\text {adiab }}=\frac{2 \sqrt{3}}{\pi} \frac{\delta}{\lambda \beta_{e}} k_{y} \Delta^{\prime}
$$

where $\Delta^{\prime}=(2 / \lambda)\left(\left(k_{y} \lambda\right)^{-1}-k_{y} \lambda\right)$ is the stability parameter for collisionless tearing modes for the equilibrium under consideration. Expression (4.18) is valid in the limit of small values of $\Delta^{\prime}$. In the absence of parallel temperature fluctuations ( isothermal limit ), the expression for the growth rate, according to Porcelli (1991), reads

$$
\gamma_{\text {isoth }}=\gamma_{\text {adiab }} / \sqrt{3}
$$

Therefore, temperature fluctuations tend to increase the growth rate. Although the expressions for the growth rates considered here are valid for the purely $2 \mathrm{D}$ case, we expect them to provide sufficiently good approximations, for the sake of the argument that follows, also in the case of oblique modes with $\left|k_{z}\right| \ll\left|k_{y}\right|$. This assumption is supported by numerical evidence (Borgogno et al. 2017).

If the Landau damping term $q_{\|_{e}}$ is included, for the magnetic equilibrium under consideration, the action of the operator $L$ in Fourier space corresponds to

$$
\mathcal{L} f_{k_{y}, k_{z}}=\frac{1}{\sqrt{\left(1-\left(\lambda / L_{x}\right) \tanh \left(L_{x} / \lambda\right)\right) k_{y}^{2}+k_{z}^{2}}}\left(-\frac{\partial A_{\| e q}}{\partial x} i k_{y}+i k_{z}\right) \check{f}_{k_{y}, k_{z}},
$$

where $A_{\| e q}(x)=-\lambda \ln \cosh (x / \lambda)$ is the equilibrium magnetic flux function. Note that, if compared with the expression for $\mathcal{L}$ in Fourier space obtained from Eq. (2.42), no Fourier transform has been carried out along the $x$ coordinate due to the choice of a non periodic equilibrium magnetic field.

The linearized electron parallel temperature (4.9) can then be written, in Fourier space, as

$$
\begin{gathered}
\frac{\partial t_{\| e_{k_{y}, k_{z}}}}{\partial t}=-\frac{2 \alpha}{\sqrt{\left(1-\left(\lambda / L_{x}\right) \tanh \left(L_{x} / \lambda\right) k_{y}^{2}+k_{z}^{2}\right.}}\left(k_{y} \frac{\partial A_{\| e q}}{\partial x}-k_{z}\right)^{2} t_{\| e_{k_{y}, k_{z}}} \\
-\frac{4}{\beta_{e}} i\left(k_{y} \frac{\partial A_{\| e q}}{\partial x}\left(-\frac{\partial^{2}}{\partial x^{2}}+k_{y}^{2}\right)+k_{y} \frac{\partial^{3} A_{\| e q}}{\partial x^{3}}-k_{z}\left(-\frac{\partial^{2}}{\partial x^{2}}+k_{y}^{2}\right)\right) A_{\| k_{y}, k_{z}},
\end{gathered}
$$

and $t_{\| e_{k_{y}, k_{z}}}, A_{\| k_{y}, k_{z}}$ are the Fourier coefficents of the perturbations of the magnetic flux function $\tilde{A}$ and of the parallel electron temperature $\tilde{t}_{\|_{e}}$, so that

$$
\tilde{A}_{\|}(x, y, z, t)=\sum_{k y, k z=-\infty}^{+\infty} A_{\| k_{y}, k_{z}}(x, t) \exp \left[i\left(k_{y} y+k_{z} z\right)\right]
$$

and

$$
\tilde{t}_{\|_{e}}(x, y, z, t)=\sum_{k y, k z=-\infty}^{+\infty} t_{\| e_{k_{y}, k_{z}}}(x, t) \exp \left[i\left(k_{y} y+k_{z} z\right)\right] .
$$

From Eq. (4.21) it follows that the first term on the right-hand side, which corresponds to the Landau damping term, vanishes at the resonant surface, which identifies the reconnection region. On the other hand, such term possesses a negative definite coefficient and tends to decrease the amplitude of the squared modulus of the Fourier coefficients $\left|t_{\| e_{k_{y}, k_{z}}}\right|^{2}$, thus leading to damping of electron temperature fluctuations. On the basis 


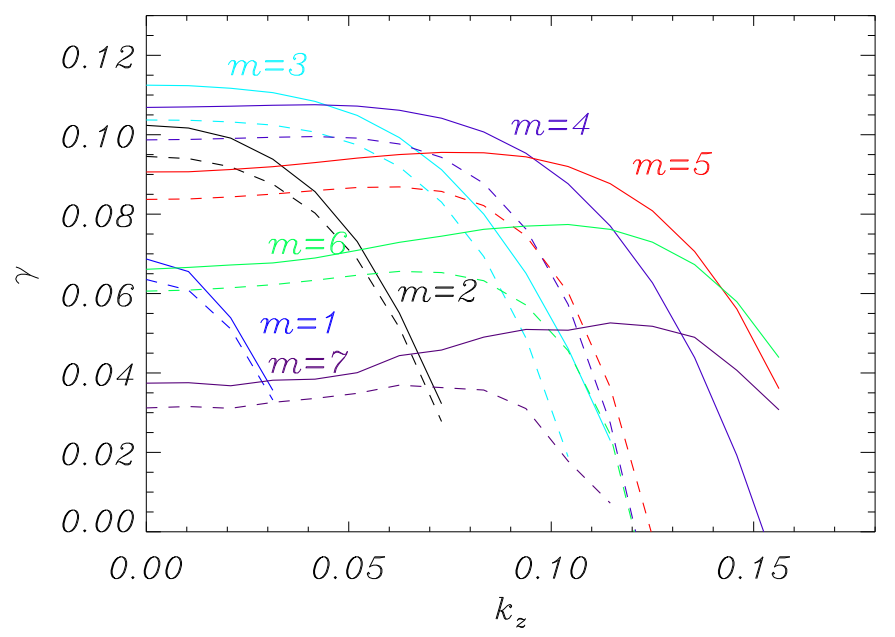

FigURE 1. Linear growth rates of reconnecting modes for simulations in the presence (dashed lines) and in the absence (solid lines) of the Landau damping term. For all modes under consideration, the Landau damping term acts to reduce the growth rate.

of the above reasoning and of relation (4.19), we expect the Landau damping term to decrease the reconnecting growth rate. This statement is confirmed by numerical simulations and illustrated in Fig. 1, where linear growth rates as function of $k_{z}$, for different values of the poloidal mode number $m$, are shown for cases including and excluding the Landau damping term. The comparison between simulations with or without the Landau damping term clearly shows that the latter reduces the linear growth rate for all modes considered. By reducing the temperature fluctuations, the Landau damping term inhibits reconnecting modes leading, for $m \geqslant 4$, to complete stabilization at sufficiently large values of $k_{z}$, where the corresponding modes in the absence of Landau damping are unstable.

We remark that the relations (4.18) and (4.19), obtained from a fluid description, can also be obtained from the formula (B71) of Zocco \& Schekochihin (2011), which accounts for the full electron kinetic response, considering the specific limits of adiabatic and isothermal electrons, respectively. Such more general formula also pertains to the small $\Delta^{\prime}$ regime and to modes with $k_{z}=0$. Denoting with $\gamma_{k i n}$ the growth rate calculated with the formula (B71) of Zocco \& Schekochihin (2011), we obtain $\gamma_{i s o t h}<\gamma_{k i n}<\gamma_{a d i a b}$, and in particular $\gamma_{k i n}=0.93 \gamma_{a d i a b}$.

In Fig. 2 we show a comparison between numerical growth rates obtained from our Landau-gyrofluid model and analytical growth rates calculated using the formula (B70) of Zocco \& Schekochihin (2011), valid for arbitrary values of $\Delta^{\prime}$. Indeed, the comparison refers to growth rates of modes with $k_{z}=0$ and values of $k_{y}$ ranging in an interval that covers both the small and the large $\Delta^{\prime}$ regimes (note that $\Delta^{\prime} \rightarrow+\infty$ as $k_{y} \rightarrow 0^{+}$). The growth rates obtained from the Landau-gyrofluid model agree well, over the whole range of $k_{y}$ considered, with the analytical growth rates derived from a formula which takes into account the full kinetic response of the electrons. We remark that, on the basis of the analysis of Fig. 1, for $k_{z}=0$, the impact of Landau damping is less pronounced with respect to modes with higher $k_{z}$, so that for purely two-dimensional disturbances, the adiabatic model could approximate the kinetic results with errors of comparable amplitude, with respect to the Landau-gyrofluid model. The advantages of including 


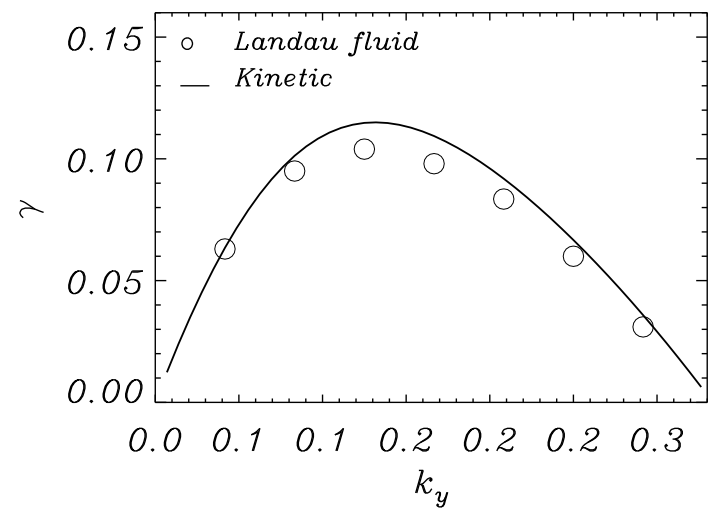

Figure 2. Comparison between growth rates obtained numerically from the Landau-gyrofluid model (circles) and analytical growth rates (solid line) obtained from the formula (B70) of Zocco $\&$ Schekochihin (2011). The growth rates refer to perturbations with $k_{z}=0$.

Landau damping are then expected to become more evident for modes with larger values of $k_{z}$.

\subsection{Nonlinear regime}

In order to investigate the influence of Landau damping on the nonlinear phase of magnetic reconnection, we analyzed the plasma dynamics induced by a magnetic perturbation of wave numbers $k_{y}=1 / 12, k_{z}=1 / 48$ in a slab with $L_{x}=46, L_{y}=12 \pi$, $L_{z}=48 \pi$. Many works in literature showed that the numerical integration of Eqs. (4.7)(4.10) in the absence of Landau damping effects is non-trivial, due to the co-existence of a broad range of time and length scales (Grasso et al. 2007; Del Sarto et al. 2003; Chacon \& Stanier 2016). In the present work, we verified that the numerical solution is even stiffer when the heat fluxes are retained. Within our third order Adam-Bashforth explicit algorithm, the time step in the nonlinear phase is indeed to be decreased, when the $q_{\|_{e}}$ field contribution is dominant, by up to two orders of magnitude with respect to the time step used in the linear phase. This makes numerical simulations very expensive in three-dimensional geometry, even when using an efficiently scalable parallel algorithm. In order to reduce the computational time, while preserving the accuracy in the spatial discretization, we adopted an alternative approach valid for single helicity problems. As described in Borgogno et al. (2005), it consists in the solution of an equivalent twodimensional problem in the new coordinates $\left(x, y_{*}\right)$, where $y_{*}=y+\left(k_{z} / k_{y}\right) z$, with a shifted equilibrium magnetic flux function $A_{\| e q}^{*}(x)=A_{\| e q}(x)-\left(k_{z} / k_{y}\right) x$.

Figure 3 shows the evolution of characteristic properties of the magnetic island with and without the Landau term versus the time normalized with respect to the corresponding growth rate. In particular, we plotted in black the logarithm of the magnetic island area, reproducing the reconnected flux at the island $X$-point, and in cyan the $X$ - point position along the $x$ direction. Note that for the nonsymmetric tearing modes the radial position of the magnetic null point varies in time because it does not coincide with a stagnation point, in contrast with the symmetric configurations. Note also that in Fig. 3 the horizontal axis indicates the time normalized by the growth rate corresponding to each simulation. Since we know from the linear analysis shown in Sec. 4.2 that Landau damping reduces the reconnection growth rate, the observation that the plots for the cases with and without Landau damping essentially overlap, in fact indicates that Landau damping slows down, with respect to the time $t$, also the nonlinear phase of the reconnection 
process. On the other hand, Fig. 3 shows that the Landau term does not affect the saturation level of the magnetic island area. We remark that two-dimensional simulations based on a hybrid model showed agreement, in terms of saturated island width, with the results of a reduced fluid model, as long as the value of the normalized $\Delta^{\prime}$ parameter did not become too small (Loureiro et al. 2013). Moreover, we can state that the saturated amplitudes measured in our simulations are comparable to the ones reported in Loureiro et al. (2013).

In Fig. 4 we consider the variation of the different terms contributing to the Hamiltonian (4.13) with respect to their initial value and normalized to the total energy at time $t=0$, for the two cases under considerations. In particular, the variations are defined as $(1 / 2) \int d^{3} x(\xi(x, y, z, t)-\xi(x, y, z, 0)) / H(0)$, where the function $\xi$ can be replaced by the different contributions to the Hamiltonian $H$. So one has $E_{p e}$ for $\xi=n_{e}^{2}$ (internal energy due to density fluctuations), $E_{T \|}$ for $\xi=t_{\|_{e}}^{2} / 2$ (parallel thermal energy), $E_{k e}$ for $\xi=\delta^{2} u_{e}^{2}$ (parallel kinetic energy), $E_{k p}$ for $\xi=\left|\nabla_{\perp} \varphi\right|^{2}$ (perpendicular plasma kinetic energy), $E_{\text {mag }}$ for $\xi=\left(2 / \beta_{e}\right)\left|\nabla_{\perp} A_{\|}\right|^{2}$ (magnetic energy). We also defined $E_{\text {landau }}=1-H(t) / H(0)=$ $(1 /(2 H(0))) \int_{0}^{t} d w \int d^{3} x t_{\|_{e}} \nabla_{\|} q_{\|_{e}}$, which corresponds to the energy dissipated via Landau damping. Note that the perpendicular thermal energy $E_{T \perp}$, corresponding to $\xi=t_{\perp_{e}}^{2}$, is neglected because of the choice of the initial conditions $\varphi(x, y, z, 0)=0, t_{\perp_{e}}(x, y, z, 0)=$ 0 , that does not allow any time variation of $t_{\perp_{e}}$, according to Eq. (4.10). We remark that, in the non-dissipative case, the percentage of total energy dissipated numerically when saturation is reached (at about $t=10.5 / \gamma$ ) is $|1-H(t=10.5 / \gamma) / H(0)| \approx 0.002$, thus just about one fifth of the variation of magnetic energy at the same time, given by $\left|E_{\text {mag }}(t=10.5 / \gamma)\right|$. By extrapolating this result to the case with Landau damping, one finds that the energy dissipated numerically is thus a small (although non-negligible) fraction of that dissipated by the Landau damping term.

Before the peak of the reconnection rate, which occurs for $\gamma t \sim 10$ as shown in Fig. 5 by the parallel electric field at the island X-point, the energy balance is similar in the two cases. In this time interval, the decrease of the magnetic energy is the same, as well as the growth of the perpendicular plasma kinetic energy that represents the dominant term of the energy balance. The main differences appear in the advanced nonlinear phase, when the process moves towards the saturation. In the Hamiltonian case, due to the formation of small scales in the fields $n_{e}, t_{\|_{e}}$ and $u_{e}$, the contributions of the terms $E_{p e}, E_{k e}$ and $E_{T \|}$ become comparable or even higher than $E_{k p}$. In particular, the internal energy due to electron temperature fluctuations represents the main sink where the magnetic energy is transferred in this phase. On the other hand, when Landau damping is retained, the contribution of all these terms is significantly lower than before and the initial energy is almost exclusively dissipated into electron heating, as observed also in Loureiro et al. (2013). We remark that no time lag is observed in our dissipative simulation between the maximum of the reconnection and dissipation rates, according to the fact that this lag is a typical feature of the weakly dissipative systems (Loureiro et al. 2013).

The spatial distributions of the fields $\varphi, n_{e}, J$ and $T_{\|_{e}}$ in the dissipationless and in the dissipative regime we considered are shown in Fig. 6 and Fig. 7, respectively. No significant differences appear in the electrostatic potential, that exhibits in both cases large-scale structures along the separatrices of the magnetic islands. The major discrepancies concern the other fields. The thin layers where $n_{e}, J$ and $T_{\|_{e}}$ are located, characteristic of collisionless reconnection (Grasso et al. 2001), are smoothed out by Landau damping. The major differences clearly involve the parallel electron temperature, whose peaks are almost twice smaller in the dissipative regime than in the dissipationless 


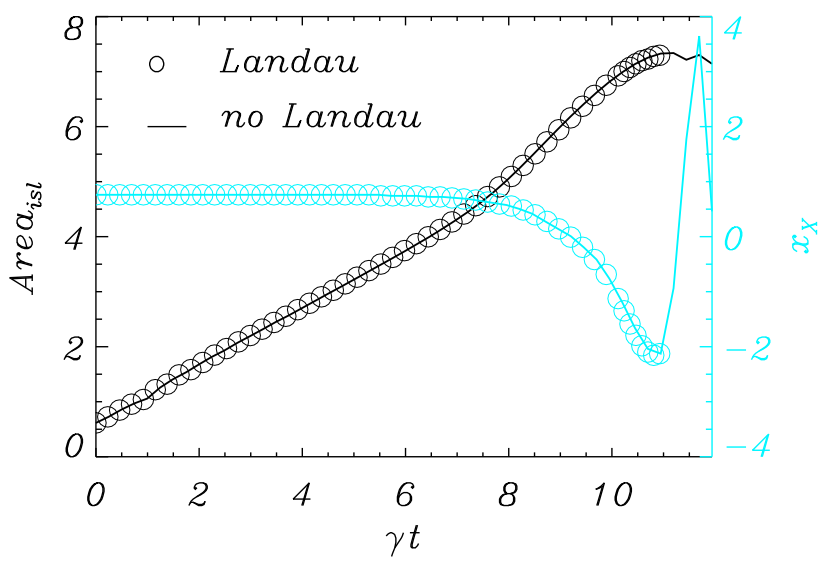

Figure 3. Time evolution of the magnetic island area (black) and the $\mathrm{x}$ coordinate of the $X$-point position (cyan) for the cases with (circle) and without (solid line) the Landau damping contribution. Each set of data is plotted versus the normalized time $\gamma t$, where $\gamma$ is the corresponding linear growth rate. Because $\gamma$ is smaller when Landau damping is taken into account, the growth of the magnetic island with respect to the time $t$ is slower in the case with Landau damping than without.
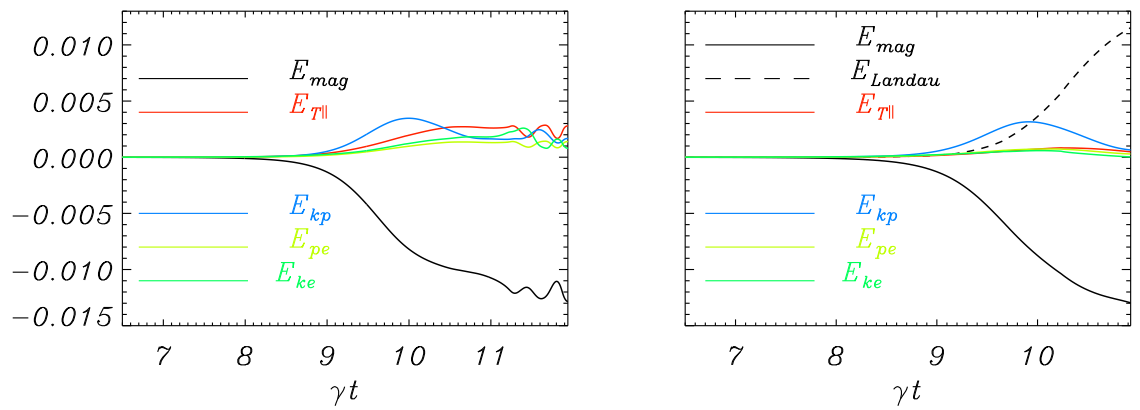

Figure 4. Time variations of the different components of the energy with respect to its initial value, divided by the total energy, for the dissipationless (left frame) and the dissipative case (right frame). The curves are plotted versus the normalized time $\gamma t$ in order to simplify the comparison between the two regimes and refer to the nonlinear phase of the reconnection process. The range of the vertical axis is the same for both the left and the right frame.

case.

\section{Conclusions}

A reduced Landau gyrofluid model for low- $\beta$ collisionless plasmas was derived and applied to a magnetic reconnection problem. The model was derived from a parent gyrofluid model by an asymptotic expansion based on a prescribed ordering and by imposing a Landau fluid closure on the electron heat fluxes. Gyro-effects were neglected for electrons but retained for the ion gyrocenters, leading to a closed six-field model. Such model generalizes the model of Schep et al. (1994) by including Landau damping closures, parallel and perpendicular electron temperature fluctuations, as well as ion 


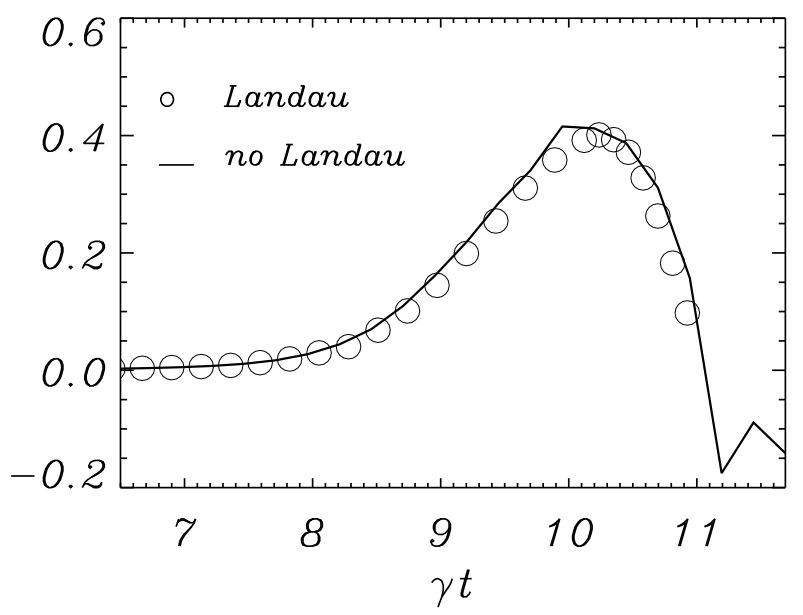

FiguRE 5. Time evolution in the nonlinear regime of the parallel electric field in the dissipative (circle) and dissipationless case (solid line) versus the corresponding normalized time.
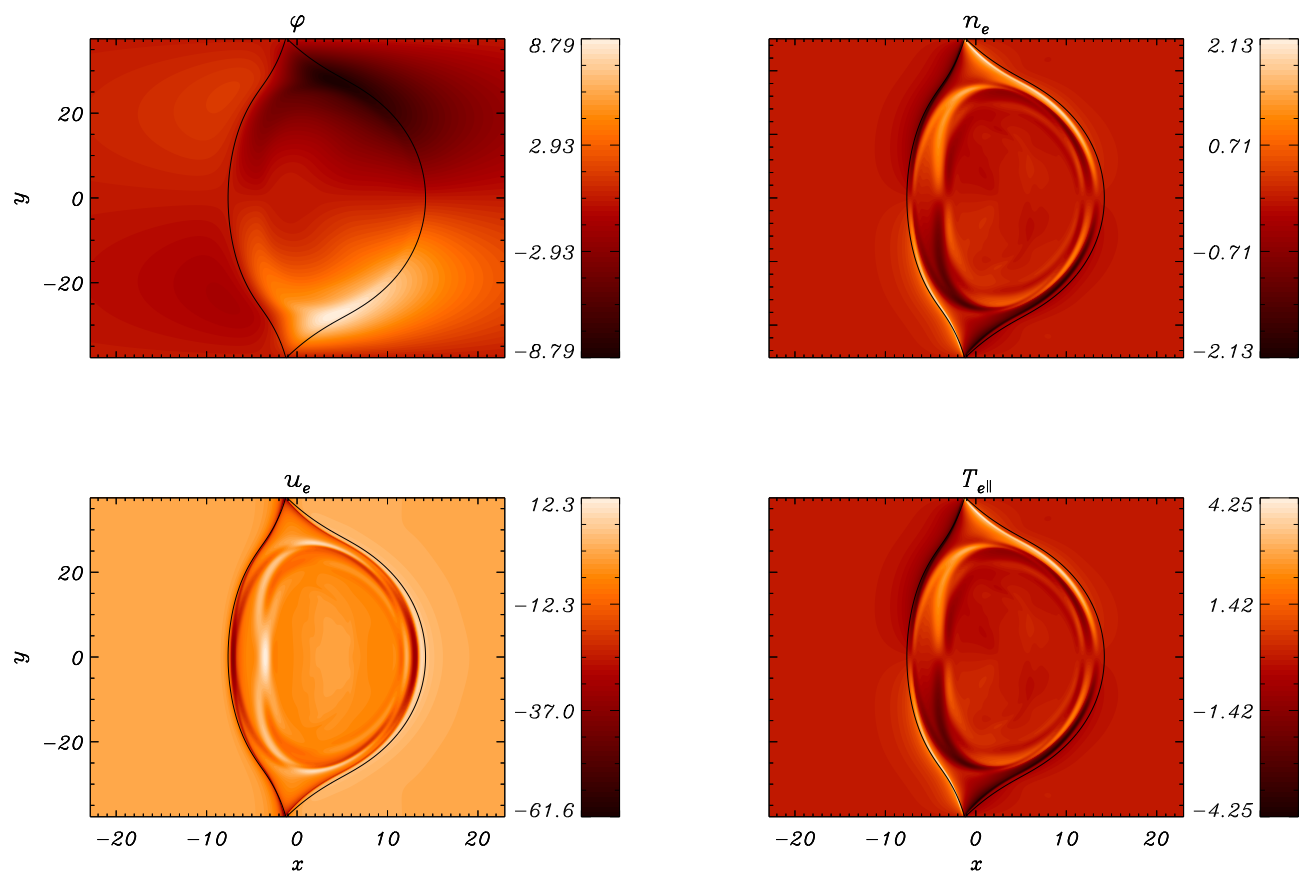

Figure 6. Contour plot of the fields $\varphi, n_{e}, u_{e}, T_{\|_{e}}$ at the peak of the reconnection rate in the dissipationless regime. The superimposed black lines map the separatrices of the corresponding magnetic island. The range of the vertical axis is the same for both left and right frames. 

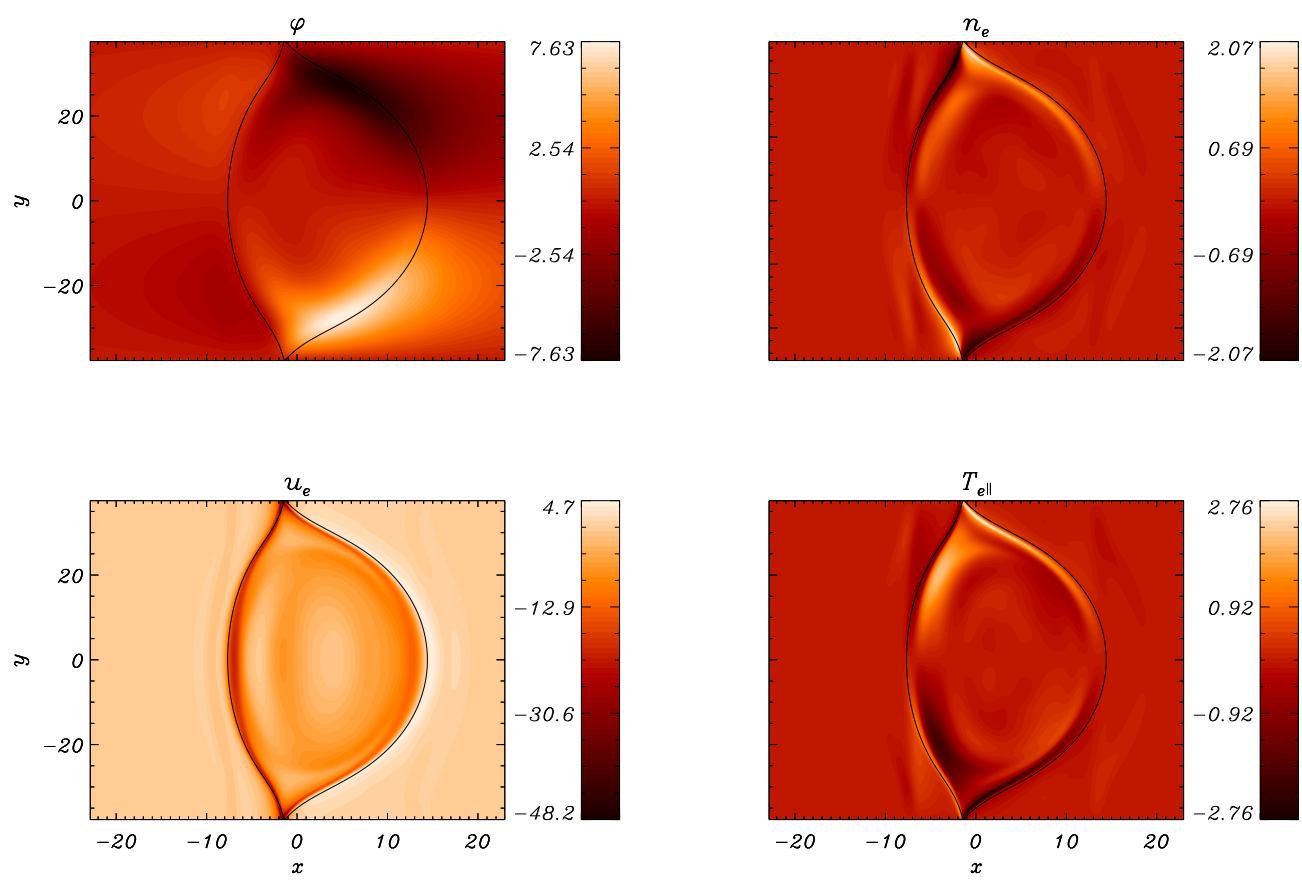

Figure 7. Contour plot of the fields $\varphi, n_{e}, u_{e}, T_{\|_{e}}$ at the peak of the reconnection rate in the dissipative regime. The superimposed black lines map the separatrices of the corresponding magnetic island. The range of the vertical axis is the same for both left and right frames.

gyrofluid perpendicular temperature fluctuations. We showed that, in the absence of Landau damping terms, the model possesses a noncanonical Hamiltonian structure. The Poisson structure is characterized by a one-parameter family of Poisson brackets. The change from the original field variables to six normal field variables suggested by the Casimir invariants, allowed to cast the Poisson bracket and the model equations in their simplest form. Four out of the six resulting evolution equations are advection equations for Lagrangian invariants. Two of such advection equations express electron entropy conservation and advection of electron perpendicular temperature fluctuations. The remaining two advection equations, on the other hand, concern two Lagrangian invariants which are linear combinations of ion gyrocenter density and perpendicular temperature fluctuations, advected by an electrostatic potential with ion gyro-radius corrections. Such advection equations appear frequently in Hamiltonian reduced fluid models, in the two-dimensional limit (see, e.g. Tassi (2017), Tassi (2015), Keramidas Charidakos et al. (2015), Hazeltine et al. (1987) and Grasso \& Tassi (2015)), although previous reduced Hamiltonian models involved only parallel temperature fluctuations.

The derived model was subsequently considered in a simplified version which neglects ion equilibrium temperature as well as ion gyrocenter density and perpendicular temperature fluctuations. In this limit, the system was trivially re-expressed in terms of particle fluid moments, yielding a generalized reduced magnetohydrodynamics model accounting for electron inertia, electron parallel and perpendicular temperature fluctuations and Landau closures for the heat fluxes. Magnetic reconnection in a 3D domain, triggered by single-helicity perturbation was then simulated numerically on the basis of such simplified version of the model. It was found that, by reducing the energy associated with 
parallel temperature fluctuations, parallel Landau damping acts to decrease the linear growth rate of reconnection. In particular, for higher $m$-modes, Landau damping can actually completely stabilize perturbations, at large enough $k_{z}$. In the nonlinear regime, Landau damping does not seem to influence the amplitude of the saturated magnetic island, which agrees with results obtained in Loureiro et al. (2013) with a hybrid model. Landau damping also tends to smoothen field gradients, in particular for the electron temperature fluctuations, that are directly affected by the Landau term. We also pointed out the absence of time lag between the maximum reconnection rate and dissipation rate observed with the Landau gyrofluid model, in contrast with what is observed with the hybrid model of Loureiro et al. (2013). This might be ascribed to a stronger dissipation induced by the terms that account for Landau damping in the Landau gyrofluid model, with respect to the actual Landau damping described by a drift-kinetic equation. The natural question arises then about whether the Landau fluid model indeed overestimates the amount of dissipated energy and what would be the consequences of considering a higher order closure.

\section{Acknowledgments}

Computations have been performed on the Mesocentre SIGAMM machine, hosted by Observatoire de la Côte d'Azur.

\section{REFERENCES}

Beer, M. A. \& Hammetт, G. W. 1996 Toroidal gyrofluid equations for simulations of tokamak turbulence. Phys. Plasmas 3, 4046.

Boldyrev, S., Horaites, K., Xia, Q. \& Perez, J. C. 2013 Toward a theory of astrophysical plasma turbulence at subproton scales. The Astrophysical J. 41, 777.

Borgogno, D., Grasso, D., Porcelli, F., Califano, F., Pegoraro, F. \& Farina, D. 2005 Aspects of three-dimensional magnetic reconnection. Phys. Plasmas 12, 032309.

Borgogno, D., Perona, A. \& Grasso, D. 2017 Test-electron analysis of the magnetic reconnection topology. Phys. Plasmas 24, 122303.

Brizard, A. 1992 Nonlinear gyrofluid description of turbulent magnetized plasmas. Phys. Fluids B 4, 1213-1228.

Camargo, S. J., Scott, B. D. \& Biskamp, D. 1996 The influence of magnetic fluctuations on collisional driftwave turbulence. Phys. Plasmas 3, 3912.

Chacon, L. \& Stanier, A. 2016 A scalable, fully implicit algorithm for the reduced two-field low- $\beta$ extended MHD model. J. Comp. Phys. 326, 763.

Comisso, L., Grasso, D., Tassi, E. \& Waelbroeck, F. L. 2012 Numerical investigation of a compressible gyrofluid model for collisionless magnetic reconnection. Phys. Plasmas 19, 042103.

Del Sarto, D., Califano, F. \& Pegoraro, F. 2003 Secondary instabilities and vortex formation in collisionless-fluid magnetic reconnection. Phys. Rev. Lett. 91, 235001.

Del Sarto, D., Califano, F. \& Pegoraro, F. 2006 Electron parallel compressibility in the nonlinear development of two-dimensional collisionless magnetohydrodynamic reconnection. Mod. Phys. Lett. B 20, 931.

Dorland, W. \& Hammett, G. W. 1993 Gyrofluid turbulence models with kinetic effects. Phys. Fluids B 5, 812-835.

Goswami, P., Passot, T. \& Sulem, P. L. 2005 A Landau fluid model for warm collisionless plasmas. Phys. Plasmas 12, 2109.

Grasso, D., Borgogno, D. \& Pegoraro, F. 2007 Secondary instabilities in two- and threedimensional magnetic reconnection in fusion relevant plasmas. Phys. Plasmas 14, 055703.

Grasso, D., Califano, F., Pegoraro, F. \& Porcelli, F. 2001 Phase mixing and saturation in Hamiltonian reconnection. Phys. Rev. Lett. 86, 5051-5054. 
Grasso, D. \& TAssi, E. 2015 Hamiltonian magnetic reconnection with parallel heat flux dynamics. J. Plasma Phys. 81, 495810501.

Hammett, G. W., Beer, M. A., Dorland, W., Cowley, S. C. \& Smith, S. A. 1993 Developments in the gyrofluid approach to tokamak turbulence simulations. Plasma Phys. Control. Fusion 35, 973.

Hammett, G. W., Dorland, W. \& Perkins, F. W. 1992 Fluid models of phase mixing, Landau damping, and nonlinear gyrokinetic dynamics. Phys. Fluids B 4, 2052.

Hammett, G. W. \& Perkins, F. W. 1990 Fluid moment models for Landau damping with application to the ion-temperature-gradient instability. Phys. Rev. Lett. 64, 3019.

Hasegawa, A. \& Mima, K. 1978 Pseudo-three dimensional turbulence in magnetized nonuniform plasma. Phys. Fluids 21, 87.

Hasegawa, A. \& Wakatani, M. 1983 Plasma edge turbulence. Phys. Rev. Lett. 50, 682.

Hazeltine, R. D., Hsu, C. T. \& Morrison, P. J. 1987 Hamiltonian four-field model for nonlinear tokamak dynamics. Phys. Fluids 30, 3204-3211.

Hazeltine, R. D., Kotschenreuther, M. \& Morrison, P. J. 1985 A Four-Field Model for Tokamak Plasma Dynamics. Phys. Fluids 28, 2466-2477.

Keramidas Charidakos, I., Waelbroeck, F. L. \& Morrison, P. J. 2015 A Hamiltonian five-field gyrofluid model. Phys. Plasmas 22, 112113.

Kim, C., Horton, W. \& Hamaguchi, H. 1993 New fluid model for the turbulent transport due to the ion temperature gradient. Phys. Plasmas 5, 1516.

Kobayashi, S., F., S., Passot, T., Laveder, D., Sulem, P., Huang, S. Y., Henri, P. \& SMETs, R. 2017 Three-dimensional simulations and spacecraft observations of sub-ion scale turbulence in the solar wind: influence of Landau damping. Astrophys. J. 839, 122.

Laveder, D., Marradi, L., Passot, T. \& Sulem, P. L. 2011 Fluid simulations of mirror constraints on proton temperature anisotropy in solar wind turbulence. Geophys. Res. Lett. 38, L7108.

Loureiro, N. F., Schekochinin, A. A. \& Zocco, A. 2013 Fast collisionless reconnection and electron heating in strongly magnetized plasmas. Phys. Rev. Lett. 111, 025002.

Marsden, J. E. \& Ratiu, T. S. 2002 Introduction to Mechanics and Symmetry. SpringerVerlag, Berlin.

Mattor, N. 1998 Collisionless fluid closure theory and the phase velocity transform. Phys. Plasmas 5, 1822.

Mattor, N. \& Parker, S. E. 1997 Nonlinear kinetic fluid equations . Phys. Rev. Lett. 79, 3419.

Morrison, P. J. 1998 Hamiltonian description of the ideal fluid. Rev. Mod. Phys. 70, 467-521.

Muraglia, M., Agullo, O., Benkadda, S., Garbet, X. \& Sen, A. 2009 Nonlinear dynamics of magnetic islands imbedded in small-scale turbulence. Phys. Rev. Lett. 103, 145001.

Ng, J., Hakim, A., Bhattacharjee, A., Stanier, A. \& Daughton, W. 2017 Simulations of anti-parallel reconnection using a nonlocal heat flux closure. Phys. Plasmas 24, 082112.

Otт, E. \& Sudan, R. N. 1969 Nonlinear theory of ion acoustic waves with Landau damping. Phys. Fluids 12, 2388-2394.

Passot, T., Henri, P., Laveder, D. \& Sulem, P. L. 2014 A fluid approach for ion scale plasmas with weakly distorted magnetic fields. Euro. Phys. J. D. 68, 207.

Passot, T. \& Sulem, P. L. 2004 A Landau fluid model for dispersive magnetohydrodynamics. Phys. Plasmas 11, 5173.

Porcelli, F. 1991 Collisionless $\mathrm{m}=1$ tearing mode. Phys. Rev. Lett. 66, 425.

Schekochinin, A. A., Cowley, S. C., W. Dorland, G. W. H., Howes, G. G., Quataert, E. \& Tatsuno, T. 2009 Astrophysical gyrokinetics: kinetic and fluid turbulent cascades in magnetized weakly collisional plasmas. Astrophys. J. Supp. 182, 310.

Schep, T. J., Pegoraro, F. \& Kuvshinov, B. N. 1994 Generalized two-fluid theory of nonlinear magnetic structures. Phys. Plasmas 1, 2843-2851.

Scott, B. 1997 Three-dimensional computation of drift Alfvén turbulence. Plasma Phys. Control. Fusion 39, 1635-1668.

ScOTt, B. 2010 Derivation via free energy conservation constraints of gyrofluid equations with finite-gyroradius electromagnetic nonlinearities. Phys. Plasmas 17, 102306.

Scotт, B. D. 2002 The nonlinear drift wave instability and its role in tokamak edge turbulence. New J. Phys. 4, 52. 
Sharma, P., Hammett, G. W., Quataert, E. \& Stone, J. M. 2006 Shearing box simulation of the MRI in a collisionless plasma. Astrophys. J. 637, 952-967.

Snyder, P. B. \& Hammett, G. W. 2001 A Landau fluid model for electromagnetic plasma microturbulence. Phys. Plasmas 8, 3199-3216.

Snyder, P. B., Hammett, G. W. \& Dorland, W. 1997 Landau fluid models of collisionless magnetohydrodynamics. Phys. Plasmas 4, 3974.

Strauss, H. R. 1976 Nonlinear, three-dimensional magnetohydrodynamics of noncircular tokamaks. Phys. Fluids 19, 134.

Sulem, P., Passot, T., Laveder, D. \& Borgogno, D. 2016 Influence of the nonlinearity parameter on the solar wind sub-ion magnetic energy spectrum: FLR-Landau fluid simulations. Astrophys. J. 818, 66.

Sulem, P. L. \& PAssot, T. 2015 Landau fluid closures with nonlinear large-scale finite Larmor radius corrections for collisionless plasmas. J. Plasma Phys. 81, 325810103.

TASsi, E. 2015 Hamiltonian fluid reductions of electromagnetic drift-kinetic equations for an arbitrary number of moments . Annals of Physics 362, 239.

TASsi, E. 2016 Hamiltonian reduced fluid model for plasmas with temperature and heat flux anisotropies. Theor. and Math. Phys. 188, 1377.

TAssi, E. 2017 Hamiltonian closures in fluid models for plasmas. Eur. Phys. J. D 71, 269.

Tassi, E., Morrison, P. J., Grasso, D. \& Pegoraro, F. 2010 Hamiltonian four-field model for magnetic reconnection: nonlinear dynamics and extension to three dimensions with externally applied fields. Nucl. Fusion 50, 034007.

Tassi, E., Morrison, P. J., Waelbroeck, F. L. \& Grasso, D. 2008 Hamiltonian formulation and analysis of a collisionless fluid reconnection model. Plasma Phys. Control. Fusion 50, 085014.

Tassi, E., Passot, T. \& Sulem, P. L. 2016 Reduced models accounting for parallel magnetic perturbations: gyrofluid and finite Larmor radius-Landau fluid approaches. J. Plasma Phys. 82, 705820601.

Thiffeault, J. L. \& Morrison, P. J. 2000 Classification and Casimir invariants of Lie-Poisson brackets. Physica D 136, 205-244.

Waelbroeck, F. L., Hazeltine, R. D. \& Morrison, P. J. 2009 A Hamiltonian electromagnetic gyrofluid model. Phys. Plasmas 16, 032109.

Waelbroeck, F. L. \& TAssi, E. 2012 A compressible Hamiltonian electromagnetic gyrofluid model. Commun. Nonlinear Sci. Numer. Simulat. 17, 2171.

Wang, L., Hakim, A. H., Bhattacharjee, A. \& Germaschewski, K. 2015 Comparison of multi-fluid moment models with particle-in-cell simulations of collisionless magnetic reconnection. Phys. Plasmas 22, 012108.

Zocco, A. \& Schekochinin, A. 2011 Reduced fluid-kinetic equations for low-frequency dynamics, magnetic reconnection, and electron heating in low-beta plasmas. Phys. Plasmas 18, 102309. 